\title{
Indicateurs de gestion et difficultés de renouvellement du " genre professionnel »
}

Vertus et limites d'une action sur les indicateurs pour transformer les pratiques professionnelles

Management indicators and the difficulties of renewing the "job genre". The virtues and limitations of using management indicators to transform occupational practices

Damien Collard et Jean-Baptiste Suquet

\section{(2) OpenEdition}

\section{Journals}

Édition électronique

URL : http://journals.openedition.org/activites/772

DOI : 10.4000 /activites.772

ISSN : $1765-2723$

Éditeur

ARPACT - Association Recherches et Pratiques sur les ACTivités

Référence électronique

Damien Collard et Jean-Baptiste Suquet, «Indicateurs de gestion et difficultés de renouvellement du " genre professionnel » », Activités [En ligne], 10-2 | Octobre 2013, mis en ligne le 15 octobre 2013, consulté le 20 avril 2019. URL : http://journals.openedition.org/activites/772 ; DOI : 10.4000/ activites. 772

\section{(c) (i) $\Theta($}

Activités est mis à disposition selon les termes de la licence Creative Commons Attribution - Pas d'Utilisation Commerciale - Pas de Modification 4.0 International. 


\title{
Indicateurs de gestion et difficultés de renouvellement du « genre professionnel » \\ Vertus et limites d'une action sur les indicateurs pour transformer les pratiques professionnelles
}

\author{
Damien Collard \\ Université de Franche-Comté,LEG - UMR 5118, Université de Bourgogne et CNRS \\ 1 rue Claude Goudimel, 25030 Besançon cedex \\ damien.collard@univ-fcomte.fr
}

Jean-Baptiste Suquet

Reims Management School, CMAC, 59 rue Pierre Taittinger - 51100 Reims

jean-baptiste.suquet@reims-ms.fr

\begin{abstract}
Management indicators and the difficulties of renewing the "job genre". The virtues and limitations of using management indicators to transform occupational practices. Based on an intervention research study on the implementation of the RATP "Bus Attitude" project and the consequent evolution of control methods, the article describes and analyses the ticket inspector "job genre". It shows how certain management indicators can play a critical role in constituting and maintaining the "job genre", sometimes even devitalizing it. The authors then question the relevance of acting on the management indicators to transform job practices and revitalize the "job genre". The article highlights the advantages and limitations of such an action and examines the role of the researcher during the intervention.
\end{abstract}

KEYWORDS

management indicators, "genre of job", intervention research

\section{Introduction}

Est-il possible de transformer les pratiques professionnelles à partir d'une action sur les indicateurs de gestion? Et peut-on, par la même, faire évoluer les «genres professionnels » (Clot \& Faïta, 2000) en vigueur dans une organisation, chaque genre étant constitutif d'une identité de métier et d'un socle de pratiques professionnelles (considérées comme légitimes par les gens du métier)?

Nous tenterons de répondre à ces questions en structurant notre propos en quatre temps. Dans une première partie, après avoir passé en revue certains travaux de recherche en sciences des organisations qui portent sur le rôle joué par les indicateurs de gestion dans la structuration du « réel » et remarqué que ces travaux n'étudient pas spécifiquement l'impact de ces indicateurs sur les pratiques de métier, nous présenterons brièvement la clinique de l'activité (à l'origine du concept de «genre professionnel») et constaterons que ce courant n'interroge pas vraiment la place que peuvent tenir certains indicateurs de gestion dans le maintien du « genre professionnel ».

Pourtant, nous entendons montrer que certains indicateurs de gestion peuvent jouer un rôle 
structurant dans la constitution et le maintien du «genre professionnel», au point de le dévitaliser ${ }^{1}$. Si cette situation n'a pas été analysée en tant que telle par les cliniciens de l'activité c'est avant tout parce que le poids joué par «l'instance impersonnelle» du métier (Clot, 2006) dans le maintien et/ou le développement du «genre professionnel» nous semble quelque peu sous-estimé au regard de la place extrêmement importante qui est accordée à la «dimension interpersonnelle ». C'est ce qui explique, de notre point de vue, le faible intérêt des cliniciens de l'activité pour les indicateurs de gestion. Cependant, dans certaines situations, ces indicateurs rendent difficile l'évolution du « genre professionnel $»^{2}$, comme l'illustre une recherche-intervention menée il y a quelques années sur la transformation du contrôle sur le réseau de surface de la RATP suite à la mise en œuvre de la démarche «Bus Attitude» (Suquet, 2008).

Après avoir présenté dans une seconde partie le contexte organisationnel dans lequel cette démarche a pris place, sa «philosophie » et ses principes d'action, mais aussi les réactions des opérationnels (agents de base et encadrement de proximité) vis-à-vis de la «Bus attitude » (réactions révélatrices du « genre du métier » de contrôleur), nous restituerons dans une troisième partie un volet de cette recherche-intervention - à savoir un travail participatif mené avec certains responsables opérationnels de la RATP pour concevoir une organisation «multimodale» de la lutte contre la fraude et de nouveaux indicateurs de gestion en vue d'accompagner la transformation des pratiques professionnelles. Enfin, dans une dernière partie, nous entamerons un retour réflexif sur cette recherche-intervention en mettant en évidence les intérêts et les limites d'une intervention sur le travail par les indicateurs de gestion, mais aussi en suggérant des pistes de prolongement de cette intervention et en revenant sur le rôle spécifique joué par le chercheur dans l'intervention.

\section{1.- La contribution des indicateurs de gestion au maintien du « genre professionnel » : un angle mort ?}

Si pour certains (consultants ou chercheurs en gestion), une action sur l'instrumentation gestionnaire, via les indicateurs de gestion, est requise pour impulser le changement organisationnel et faire évoluer les pratiques des acteurs ${ }^{3}$, pour d'autres auteurs (spécialistes des sciences des organisations ou des sciences du travail) la réponse est moins tranchée, du fait du couplage flou existant entre les prescriptions organisationnelles et les pratiques professionnelles.

\section{1.- Le rôle joué par les indicateurs de gestion dans la structuration du « réel » : le prisme des sciences des organisations}

Pour les sciences des organisations, les indicateurs de gestion contribuent à stabiliser l'ordre

1 Selon la clinique de l'activité, le « genre professionnel » est vivant quand il est nourri par des controverses de métier. C'est un signe de sa vitalité. A contrario, l'absence de controverse a pour effet de dévitaliser le « genre professionnel».

2 La conséquence d'une absence de controverse de métier est, à court terme, une dévitalisation du « genre professionnel » et, à long terme, une sclérose.

3 Cette littérature (normative et prescriptive), à destination principalement des managers en quête de recettes, souffre de deux point faibles. Le premier est que le pilotage du changement est très (trop) souvent pensé de manière exclusivement descendante (démarche top down), faisant la part belle aux décideurs (considérés comme rationnels et éclairés), et minorant le rôle joué par les opérateurs et par l'encadrement intermédiaire dans les processus de changement et dans la transformation des pratiques professionnelles. Le second réside dans le faible intérêt, pour ne pas dire dans l'ignorance (voire le mépris) pour le travail concret des opérateurs - appréhendé généralement comme une source de résistance au changement - et pour les compétences mobilisées par ces derniers dans leur activité (ce qui n'empêche pas, paradoxalement, ces mêmes auteurs d'invoquer le développement des compétences comme une solution pour accompagner le changement organisationnel). 
organisationnel et à structurer les pratiques professionnelles. Ils constituent également des outils d'aide à la décision. Pour Jean-Claude Moisdon, ces indicateurs, que l'on retrouve dans tous les domaines de la gestion (comptabilité, contrôle de gestion, gestion des ressources humaines, gestion de production, etc.), sont des outils de gestion qui permettent aux différents acteurs (en premier lieu aux dirigeants et à l'encadrement) d'investiguer le fonctionnement organisationnel, de piloter les mutations organisationnelles et d'explorer des situations nouvelles (Moisdon, 1997). Pour Philippe Lorino, un indicateur est «une information devant aider un acteur, individuel ou plus généralement collectif, à conduire le cours d'une action vers l'atteinte d'un objectif ou devant lui permettre d'en évaluer les résultats» (Lorino, 2003a, p. 131). Pour autant, ces indicateurs sont loin d'être neutres et impartiaux, comme le soulignent plusieurs courants de recherche.

Dans le domaine de la comptabilité et du contrôle de gestion, un certain nombre de chercheurs anglo-saxons soulignent ainsi que les techniques et autres indicateurs de gestion contribuent avant tout à légitimer l'ordre social existant (Brosnan, 2005) et à reproduire certaines visions de l'organisation (Bloomfield \& Vurdubakis, 1997; Colville, 1981; Nahapiet, 1988). Les indicateurs de gestion permettraient notamment aux dirigeants de justifier leurs décisions et de véhiculer une certaine idéologie managériale (Brosnan, 2005).

Dans la même veine, des travaux menés en sociologie de la gestion soulignent la dimension politique de ces indicateurs et leur ancrage idéologique (Boussard, 2008; Boussard \& Maugeri, 2003). Certains indicateurs sont qualifiés de «prégnants» par Boussard (1999) parce qu'ils structurent le mode de fonctionnement de l'organisation, les discours et les pratiques des différents acteurs. Ils «mettent en scène, en les symbolisant, des règles sociales légitimes. Ils permettent aux règles de prendre corps, d'être visibles. Ils jouent le rôle d'étendards avec lesquels une organisation ou des groupes d'acteurs revendiquent et assoient leurs positions. Les indicateurs de gestion seraient dans cet esprit essentiellement liés à une régulation sociale des organisations » (Boussard, 2001, p. 534).

Des travaux désormais classiques menés en sciences de gestion (en France) au sein du $\mathrm{CRG}^{4}$

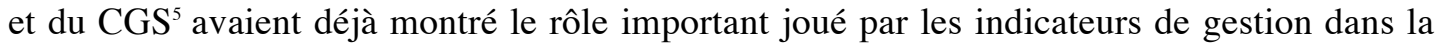
régulation des rapports sociaux et le maintien de la cohérence organisationnelle (Berry, 1983 ; Boigne, Moisdon, \& Tonneau, 1986 ; Riveline, 1981). Ainsi, pour Riveline (1981) et Berry (1983), ces indicateurs constituent des «abrégés » qui permettent aux différents acteurs de consolider leurs positions, d'évaluer rapidement une situation et de justifier plus facilement leurs choix. Pour Boigne, Moisdon et Tonneau (1986), dans certaines situations, ces indicateurs, bien que simplistes et partiels, contribuent à produire des représentations partagées et, ce faisant, facilitent la coordination et les prises de décision.

Dans le même temps, ces indicateurs sont porteurs d'effets pervers: mise en place d'automatismes de décision, baisse de la vigilance, rigidité des comportements, résistances au changement, aveuglement collectif, etc. Par ailleurs, ces mêmes chercheurs insistent sur l'ancrage culturel de ces indicateurs. À l'instar de Simondon qui s'efforce de réconcilier technique et culture dans son ouvrage "Du mode d'existence des objets techniques» (Simondon, 1958), Moisdon réintègre les outils de gestion dans la culture (Moisdon, 1997). Les indicateurs de gestion véhiculent en effet certaines «visions du monde». Ils sont souvent porteurs d' «évidences » et d'idées qui sont partagées dans l'organisation, lesquelles trouvent leurs fondements dans la culture ${ }^{6}$ et l'histoire de l'organisation (Berry, 1983).

Si certains des chercheurs cités adoptent une posture critique et ne se soucient guère

4 Centre de recherche en gestion de l'École polytechnique. A noter que les deux auteurs de ce texte sont d'anciens membres du CRG.

5 Centre de gestion scientifique de l'École des mines de Paris.

6 Pour Michel Berry, la culture constitue «l'ensemble des évidences qui s'imposent sans même qu'elles soient nécessairement explicitées » (Berry, 1983, p. 23). 
d'accompagner les acteurs de terrain dans leurs réflexions (c'est le cas des chercheurs anglosaxons en comptabilité et en contrôle de gestion mentionné précédemment ainsi que de la plupart de ceux qui se réclament de la sociologie de la gestion), il n'en va pas de même des membres fondateurs du CRG et du CGS qui font de l'intervention sur et par les indicateurs de gestion un passage obligé pour à la fois produire des connaissances sur le fonctionnement des organisations et accompagner les mutations organisationnelles. Dans la mesure où, dans bien des situations, ces indicateurs structurent les représentations et les comportements humains, qu'ils engendrent des décisions qui échappent à la volonté des hommes (Berry, 1983), et qu'ils sont sources de dysfonctionnements, changer ces indicateurs ou les faire évoluer constitue une des finalités de l'intervention.

Il y a ici une tension structurante du débat sur les indicateurs de gestion, dont les travaux de Philippe Lorino témoignent bien et indiquent en même temps une piste prometteuse quant au lien entre indicateurs et travail. Puisque les indicateurs participent d'un «système de significations qui fondent socialement l'organisation» (Lorino, 2003b), il convient justement d'adopter une perspective sémiotique et pragmatique (Lorino, 2005) : plutôt que d'entériner leur domination sur les acteurs, mener l'enquête avec les acteurs sur ces indicateurs, de manière à (re)travailler leur interprétation et transformer l'action collective.

La plupart des chercheurs se réclamant d'une action sur les indicateurs ont néanmoins constaté que certains de ces indicateurs étaient solidement arrimés, qu'ils présentaient, certes des inconvénients, mais aussi des avantages pour les acteurs impliqués, et que trouver des indicateurs de gestion alternatifs n'était pas chose simple (Berry, 1983 ; Boigne, Moisdon, \& Tonneau, 1987) ${ }^{7}$. Moisdon reconnait, à la suite d'une série d'interventions menées dans le contexte hospitalier, que si les outils de gestion génèrent de nouveaux savoirs, le système dans son ensemble n'en est pas moins « étrangement stable» (Moisdon, 2005).

C'est pour cela que les méthodes d'intervention développées au sein de ces deux centres de recherche, qui visent notamment à faire évoluer l'instrumentation gestionnaire en vigueur, reposent avant tout sur l'existence d'une «demande », sur une collaboration étroite avec les acteurs de terrain, et sur une investigation en profondeur (des organisations concernées) par les chercheurs qui s'efforcent de croiser différentes méthodes (observation participante, entretiens semi-directifs, analyse documentaire, etc.). Il s'agit là de pré-requis pour accompagner les acteurs de terrain dans leurs réflexions, faire évoluer une situation bloquée et desserrer l'étau créé notamment par certains indicateurs de gestion.

Cependant, ces différents travaux de recherche n'étudient pas spécifiquement le rôle joué par les indicateurs de gestion dans le maintien et le développement des pratiques de métier, même si ces indicateurs peuvent avoir pour effet le renforcement des identités professionnelles pour Berry (1983), la légitimation des pratiques de certains groupes sociaux pour Boussard (1999), et la production de nouveaux savoirs professionnels pour Moisdon (2000). Si tel est le cas, c'est parce que les auteurs cités, chercheurs en gestion ou sociologues des organisations, s'intéressent avant tout à l'action collective organisée et finalisée ${ }^{8}$. Pour autant, les chercheurs qui se réclament de la recherche-intervention n'ignorent pas les pratiques de métier des différents acteurs et utilisent justement les nombreux obstacles rencontrés dans le cadre de la recherche-intervention pour faire évoluer ces pratiques, notamment via l'implémentation dans l'organisation de nouveaux outils et règles de gestion, comme des révélateurs des dynamiques organisationnelles. Ainsi, dans le cadre de l'hôpital, Jean-Claude Moisdon préconise de prendre en compte les règles de métier quand on conçoit des règles de gestion, car ces dernières doivent (en théorie) satisfaire aux

7 Nous avons nous-mêmes fait ce constat à travers les recherches que nous avons menées à la RATP (Suquet, 2008) et à la SNCF (Collard, 2010).

8 Ou à ceux qui sont censés l'organiser. Ainsi Philippe Lorino centre-t-il le plus souvent son propos sur les «managers » ou les « dirigeants » (voir par exemple Lorino, 1995). 
prérequis professionnels (Moisdon, 2008). Mais la connaissance du «travail réel», de « l'activité », ou encore de l'exercice au quotidien du métier, sont utilisés comme un moyen d'accéder à une meilleure compréhension des dispositifs et outils de gestion, et ne sont pas interrogés en tant que tels. D'où l'intérêt de se tourner vers la clinique de l'activité.

\section{2.- L'analyse de l'activité et le « genre professionnel » : le prisme de la clinique de l'activité}

La clinique de l'activité est une méthode qui vise à produire des connaissances sur l'activité tout en la transformant, son objet étant le développement de l'activité et ses empêchements (Clot, 2004) $)^{9}$. L'activité est pensée en relation avec la notion de «métier ${ }^{10}$ qui est appréhendée à plusieurs niveaux. Le métier est à la fois personnel, interpersonnel, transpersonnel et impersonnel (Clot, 2006). Personnel parce qu' intime et incorporé » (Clot, 2006, p. 167). Interpersonnel «car il ne saurait exister sans destinataire 》(Clot, 2006, p. 167). Transpersonnel en tant que «répondant collectif de l'activité personnelle »(Clot, 2006, p. 167). Il s'agit du «genre du métier» ou «genre professionnel» - ensemble d'«obligations partagées qui permettent de travailler malgré tout, parfois malgré l'organisation du travail» (Clot \& Faïta, 2000, p.9) - qui s'intercale entre la tâche (prescrite) et l'activité (individuelle). Impersonnel en tant que « tâche ou fonction prescrite par l'organisation du travail » (Clot, 2006, p. 167). Pour la clinique de l'activité, le métier est vivant quand ces différentes instances sont en tension et que cette tension est créatrice et source de développement (Kostulski, lot, Litim, \& Plateau, 2011). A contrario, quand cette tension n'est plus créatrice, l'activité (individuelle et collective) est entravée dans son développement et le métier tend à se figer, par exemple, en cas de «déréalisation de l'impersonnel » ou de «nécrose du transpersonnel (Kostulski et al., 2011, p. 134). L'une des finalités de l'intervention en clinique de l'activité est alors de revitaliser le "genre professionnel » grâce à un travail de co-analyse des pratiques professionnelles avec les acteurs de terrain (les représentants du métier). Il s'agit là d'un préalable avant toute action qui porterait sur la «dimension impersonnelle » du métier et qui aboutirait à des propositions de changement au niveau de l'organisation du travail et des prescriptions organisationnelles.

Même si les indicateurs de gestion ne constituent pas un objet de réflexion (en soi) pour les cliniciens de l'activité, ils ne sont cependant pas absents de leurs préoccupations, ne serait-ce que parce qu'ils s'intéressent à l'instance impersonnelle du métier. La prise en compte de la dimension impersonnelle du métier dans l'analyse suppose en effet de s'intéresser à la conception du travail, à l'organisation des tâches et aux prescriptions organisationnelles, des prescriptions qui apparaissent souvent sous la forme de standards de performance cristallisés dans un certain nombre d'indicateurs (le nombre de voitures vendues pour un commercial, de pièces produites pour un opérateur, de procès-verbaux réalisés pour un contrôleur, etc.). Les indicateurs de gestion sont donc partie intégrante de l'instance impersonnelle du métier. Une réflexion sur ces indicateurs apparaît dès lors incontournable quand on s'intéresse au métier appréhendé dans sa dynamique propre, notamment à travers les tensions qui existent entre les instances impersonnelle et transpersonnelle du métier. Pour la clinique de l'activité, ces tensions sont créatrices quand les «formes prescriptives que les travailleurs s'imposent pour pouvoir agir» (Clot \& Faïta, 2000, p. 11) - et qui sont constitutives du «genre professionnel» (autrement dit de l'instance transpersonnelle du métier) - viennent constamment «rafraîchir » les prescriptions ou bien les contourner» (Clot \& Faïta, 2000,

9 Signalons d'emblée, que dans le cadre de cette recherche, il n'a pas été possible de déployer l'arsenal méthodologique propre à la clinique de l'activité. Cependant, la notion de « genre professionnel », empruntée à la clinique de l'activité, étant au cœur de nos réflexions, il nous a semblé indispensable de présenter ce courant de pensée et la manière dont elle envisage le «métier ».

10 Cependant, «Métier et activité ne sont pas synonymes. «Si le métier équipe l'activité, en retour elle le modifie (Clot, 2008, p. 261).» (Kostulski et al., 2011, p. 133). 
p. 13). Le développement de l'instance impersonnelle du métier dépend ainsi de la «vitalité $d u$ transpersonnel »(Kostulski et al., 2011, p. 134). A contrario, la «dégénérescence du transpersonnel » a pour effet de figer l'instance impersonnelle, et en définitive de dévitaliser le métier.

Dans l'analyse des rapports entre les instances transpersonnelle et impersonnelle du métier, une autre situation peut se présenter, celle de la «mainmise» de la dimension impersonnelle (via certains indicateurs de gestion) sur la dimension transpersonnelle, situation qui peut induire des difficultés de renouvellement du «genre professionnel » quand ce dernier n'est plus nourri par des controverses de métier. Nous allons illustrer ce cas de figure à partir d'une recherche-intervention menée à la RATP, dans le cadre d'une thèse de doctorat en gestion (Suquet, 2008), qui a porté sur les transformations de la lutte contre la fraude sur le réseau de surface. Nous montrerons notamment que l'emprise de l'instance impersonnelle sur l'instance transpersonnelle, ainsi que les difficultés de renouvellement du «genre professionnel» qui s'en suivent, sont alimentées tant par l'encadrement que par les opérateurs (ici les contrôleurs).

\section{2.- La difficile transformation du contrôle sur le réseau de surface de la RATP}

\section{1.- Une demande d'accompagnement de la mise en œuvre de la Bus Attitude}

L'un des auteurs s'est vu adresser une demande par la RATP en 2004, qui s'est traduite concrètement par une convention CIFRE soutenant son travail de thèse (Suquet, 2008). L'objet en était l'accompagnement des transformations de la lutte contre la fraude sur le réseau de surface (bus et tramway). Le ressort de cette recherche se trouvait dans la difficulté éprouvée par les membres de la Régie à articuler «lutte contre la fraude » et «relation de service ». Cette demande assez large prenait sens par rapport à un contexte particulier : la mise en ouvre de la Bus Attitude (BA), en particulier au sein du Contrôle Service Bus (CSB), entité rattachée au département BUS ${ }^{11}$.

La BA avait pour finalité de promouvoir les conditions d'une «bonne» relation entre la RATP et ses usagers sur le réseau de surface. Le «projet BA » consistait en une tentative du département BUS de modifier le comportement des voyageurs à bord des bus, notamment dans leur respect des règles de paiement, et de réinstaller les machinistes-receveurs comme «maître à bord ». L'entreprise entendait ainsi lutter contre les incivilités, la fraude et les agressions (endémiques dans les bus depuis de nombreuses années). Quant au directeur du CSB, il entendait s'appuyer sur la BA pour changer la «culture des contrôleurs » et les faire évoluer vers un rôle qui se rapprocherait de celui d'un «VRP de titres longs » (il entendait par là les abonnements) ${ }^{12}$. Cependant, selon lui, la plupart des contrôleurs étaient opposés à une telle évolution. Il confia donc au jeune chercheur une première mission : celle de rendre compte (observer et proposer une explication) des difficultés de déploiement de la BA sur le terrain.

11 Le CSB est l'entité en charge de la lutte contre la fraude sur le réseau de surface de la RATP. Ses quelques 800 agents (au moment de l'enquête) font partie du département BUS.

12 A noter que les contrôleurs sont une ressource stratégique pour la RATP parce que le contrôle se situe à l'intersection entre deux exigences dont la compatibilité n'est pas évidente : l'amélioration de la relation de service et la baisse de la fraude. 


\section{Méthodologie}

La démarche a été de suivre les principes d'une recherche ethnographique de type clinique (Berry, 1986, 1995) pour assurer une présence forte sur le terrain tout en se positionnant par rapport à la demande de l'institution.

La démarche clinique présente la caractéristique de travailler à partir du sens donné par les acteurs aux situations rencontrées, dans leur singularité et à partir de cette singularité. Adopter une telle démarche suppose de faire preuve d'un certain «opportunisme méthodique» (Girin, 1989), dans le sens où «le travail de terrain n'est pas mené suivant un protocole établi par avance, mais s'ajuste à des circonstances, nécessités, opportunités, impossibilités, négociations, etc., qui peuvent parfois l'infléchir; et qui admet que les autochtones, ceux du terrain, ont leur mot à dire sur son déroulement» (Girin, 1986, p. 1).

La demande initiale du directeur du CSB (à savoir rendre compte des difficultés de déploiement de la BA auprès de ses agents de contrôle) a conduit tout d'abord le chercheur à privilégier une observation et une analyse fines des pratiques des agents de contrôle et de l'encadrement sur 7 mois : suivis des agents sur le «terrain» (18 demi-journées), en formation (13 formations suivies), immersion dans un bureau de formateurs au CSB, entretiens avec les encadrants du CSB et plus largement avec les acteurs de la lutte contre la fraude (40 entretiens).

Ensuite, le directeur du CSB a demandé au chercheur de participer pendant 9 mois à 21 séances de travail sur l'organisation de la lutte contre la fraude dans la perspective de la mise en place d'un contrôle multimodal (réunissant les trois réseaux : Métro, RER, BUS). Cette observation participante a permis de donner à voir de façon plus approfondie le travail d'encadrement et de production des indicateurs de gestion et des processus de planification, et la place qui revenait à la BA dans la nouvelle organisation de la lutte contre la fraude (cf. partie 3).

Ainsi, cette intervention n'a pas été pensée de façon systématique a priori; mais nous essayons justement d'en faire un récit critique pour en faire apparaître rétrospectivement les éléments déterminants (dans les choix opérés par le chercheur comme dans les effets de son intervention). La mise en ouvre de la BA nécessitait une modification importante de l'organisation du travail et une transformation des pratiques professionnelles. Il était en effet demandé aux contrôleurs de sortir d'une logique principalement centrée sur la répression pour se préoccuper en premier lieu de «pédagogie». Les enjeux de satisfaction et de fidélisation des «clients» faisaient également partie de l'horizon du projet, et devaient se traduire par une démarche commerciale d'écoute et de conseil vis-à-vis des clients (actuels ou potentiels, puisque les contrôleurs rencontraient au cours de leur travail des voyageurs en règle comme en infraction). Ainsi, en ce qui concerne le contrôle, la $\mathrm{BA}$ confirmait le choix d'un certain nombre de principes élaborés et testés en amont, à plus petite échelle, à travers les Nouveaux Services Tram 1 et 2 (NST 1 et NST 2) et le Service De Ligne (SDL) : des expérimentations initiées quelques années auparavant et pérennisées depuis lors sur leurs lignes respectives (T1, T2 et TVM), dans lesquelles on trouvait déjà une organisation du travail favorisant une approche pédagogique et commerciale du contrôle. Concrètement, cela signifiait : des équipes plus petites (deux à quatre personnes au lieu le plus souvent d'une dizaine sur les lignes équivalentes) ; un contrôle itinérant (à bord du bus pendant que celui-ci roule) ou à la montée, de façon à être plus souple que lors d'un contrôle en « chute » (i.e. à la descente des voyageurs, au moment où l'infraction est déjà commise); une focalisation moindre sur le procès-verbal, au profit par exemple d'une incitation à valider ou à acheter un ticket, etc. La BA se présentait ainsi comme une occasion unique de tester à grande échelle l'efficacité et la pertinence d'une activité de contact alternative puisque le directeur du CSB décida de manière unilatérale que tous les contrôleurs devaient y consacrer $50 \%$ de leur temps de travail (les $50 \%$ restant étant consacrés à du contrôle traditionnel). Il s'agissait par ce projet de déployer sur le réseau de surface des équipes de taille réduite ayant pour rôle de susciter la civilité à bord du bus, d'améliorer la qualité de service et de fidéliser les clients. 
Cependant, deux différences de taille sont à souligner entre la BA et les expérimentations précédemment décrites. D'une part, tandis que ces dernières reposaient sur le volontariat (les agents étaient en effet volontaires pour tester de nouvelles pratiques professionnelles et adhéraient aux valeurs sous-jacentes à ces démarches), la BA s'imposait à tous les agents du CSB (volontaires ou non). D'autre part, tandis que la spécialisation géographique était une caractéristique importante des NST 1 et 2 et du SDL (elle permettait de familiariser sur une même ligne les agents à leur public), dans le cadre de la BA les agents étaient amenés à changer régulièrement de ligne et d'équipe (la mobilité était donc de mise).

L'immersion du jeune chercheur au sein du CSB lui a permis d'établir une correspondance entre des techniques de contrôle et des pratiques discursives, et de constater la prégnance du contrôle répressif, tant au niveau des pratiques que des indicateurs de gestion: le taux de fraude et le nombre de PV réalisés sont notamment apparus comme des indicateurs prégnants. Les indicateurs se sont ainsi révélés au cours de l'enquête être l'un des facteurs sur lesquels il était possible d'agir et, à ce titre, ils constituaient une opportunité pour le chercheur. Par ailleurs, l'enquête a permis de mettre en évidence deux «philosophies » du contrôle : d'un côté le « contrôle répressif », constitutif du « genre du métier » de contrôleur, tel qu'il s'est constitué historiquement à la RATP ; de l'autre le «contrôle attentionné », au fondement des NST 1 et 2, du SDL, et de la BA. Concernant le «contrôle répressif », trois facteurs expliquent son ancrage fort dans l'organisation: tout d'abord, le fait que les contrôleurs sont chargés de la lutte contre la fraude, et donc selon eux des «fraudeurs $»^{13}$ (l'attention portée aux autres voyageurs, ceux qui sont en règle, étant moindre puisqu'il s'agit avant tout de «nettoyer» les bus...) ; ensuite, le poids du PV, le nombre de PV dressés étant pour la plupart des contrôleurs et des encadrants de proximité l'étalon par rapport auquel s'évalue le travail réalisé dans la journée (voir encadré ci-dessous); enfin, la taille et la composition de l'équipe, les contrôleurs du CSB ayant tendance à associer l'efficacité de leur action à la composition plus ou moins masculine de leur équipe (« une femme équivaut, pour le contrôle, à la moitié d'un homme!» est une phrase qui a été souvent entendue par le chercheur...) et à sa taille (un effectif conséquent permettrait de resserrer les «mailles du filet » sans se mettre en danger...).

\section{La prégnance de la verbalisation dans les pratiques professionnelles}

La verbalisation peut prendre deux formes principales. Dans le premier cas, le contrevenant accepte de régler immédiatement au contrôleur le montant légalement fixé pour réparation. Le contrôleur ne relève alors pas son identité et lui délivre simplement une quittance de paiement $(\mathrm{QP})$. Dans le cas contraire, le contrôleur relève alors son identité et rédige un procès-verbal (PV) dont il lui remet un double. Le contrevenant dispose d'un certain temps pour s'acquitter du règlement. Il existe également la possibilité pour le contrôleur de recourir au «sans-suite», un imprimé qui officialise l'indulgence du contrôleur, ou encore au forfait de régulation, solution qui consiste à décider de façon discrétionnaire du montant que le contrevenant devra acquitter.

Au CSB, le PV occupe la première place. Le «sans-suite» comme le forfait de régulation sont statistiquement négligeables dans la pratique des contrôleurs. Pour le contrôleur, l'efficacité de son action passe principalement par le PV, signe le plus évident de son utilité. Il est également valorisé de «faire une QP », car le montant de l'amende est encaissé directement. Inversement, il est mal venu pour un contrôleur de recourir trop fréquemment au « sans-suite » ou au forfait de régulation, car cela peut indiquer un manque de fermeté (absence de sanction ou sanction moindre).

Par ailleurs, un contrôleur a des objectifs à atteindre. En tout premier lieu ${ }^{14}$, un certain nombre

13 Signalons que les plus durs d'entre eux (un indice en étant la violence dont ils font preuve) donnent le plus de prestige aux agents qui les verbalisent.

14 Les contrôleurs du CSB ont également d'autres objectifs, mais qui sont apparus très clairement secondaires dans leurs préoccupations, comme dans celle de leur encadrement d'ailleurs. D'une part, pour la hiérarchie de proximité, il était important d'atteindre un certain taux de contrôle des voyageurs sur les lignes du secteur, car 
«d'affaires » réalisées dans une journée de travail (une moyenne de 5 par jour en 2004) ${ }^{15}$. Tant la hiérarchie que les collègues sont attentifs à ce critère : s'il est atteint par chacun, le CSB atteint alors un niveau de production globale qui donne lieu à une prime collective annuelle.

Cet ensemble de facteurs fait du nombre de PV dressés par un agent au cours d'une journée de travail le signe principal du travail réalisé. Cet accord autour de la verbalisation donne un contenu, indiscutable parce qu'indiscuté, à l'activité du contrôle répressif, et vient consolider le «genre du métier » de contrôleur.

Cette esquisse rapide et contrastée du paysage du contrôle, au moment de l'arrivée sur le terrain, permet de souligner l'importance que revêtait la BA, et du même coup de mieux saisir la demande des commanditaires de la thèse. La BA n'était pas simplement un projet d'envergure, elle était une interpellation par la direction de la RATP du métier de contrôle. Il était donc intéressant d'étudier les effets de cette démarche sur le «genre professionnel » en vigueur.

\section{2.- Les réactions à la $\mathrm{BA}$ comme symptôme des difficultés de renouvellement du « genre professionnel »}

Dans l'esprit de la direction, la BA n'aurait pas dû nécessairement constituer un blocage. On l'a vu, les expérimentations menées s'étaient pérennisées, ce qui laissait penser à une simple généralisation réalisée auprès des 800 agents du CSB. C'est d'ailleurs ce qui était en cours lors de l'arrivée du chercheur sur le terrain, après un test de quelques mois réalisé au sein de l'un des secteurs.

Pourtant, très rapidement, une opposition assez massive est apparue au sein du CSB à propos de la BA. Trois aspects nous semblent notables dans ce que l'observation a fait ressortir. Tout d'abord, les conditions dans lesquelles le déploiement à grande échelle de la BA s'est déroulé - notamment le fait d'imposer ce projet à tous les agents (volontaires ou non) et de les obliger à pratiquer la BA sur plusieurs lignes au sein d'équipes sans cesse renouvelées - a eu pour effet de démotiver les agents (la BA leur est apparue comme vide de sens). Ensuite, le rejet était largement partagé au sein de l'unité, et les lignes de partage, qui existaient néanmoins, ne reprenaient pas le clivage hiérarchique auquel on pourrait s'attendre s'agissant d'un changement organisationnel.${ }^{16}$ Enfin, la BA était surtout victime de son affiliation au contrôle attentionné, autrement dit était perçue comme un «challenger » du contrôle répressif.

En effet, que ce soit en suivant les équipes de contrôle dans leur travail (dans sa version répressive ou attentionnée, lors des missions BA), les agents en salle de formation, ou même l'encadrement du CSB, la réponse était souvent identique : "la BA ça ne marche pas ». Cette opposition s'est faite d'autant plus forte à mesure que les arrêts maladie se multipliaient. Ainsi, un agent absent, de préférence lorsqu'il était censé «être en BA », c'était un agent qui devait être remplacé par un collègue pour constituer les équipes BA (le projet d'entreprise était bien entendu prioritaire). En résultait une rupture de l'équilibre souhaité par la direction entre BA et contrôle classique, qui n'allait pas sans accentuer la

il s'agissait d'un engagement vis-à-vis de l'autorité de régulation des transports, le STIF. Cet objectif n'a que rarement été mentionné par les agents eux-mêmes, ce qui est cohérent avec le fait que le vrai travail était pour eux lié aux passagers en infraction. Par ailleurs, les contrôleurs devaient respecter dans le cadre de leur contrôle, un certain nombre de principes relationnels : courtoisie, écoute, apparence. Le respect de ces principes était contrôlé par la technique du «client mystère ».

15 Une affaire, dans le jargon des contrôleurs de la RATP, est un PV ou bien une quittance de paiement.

16 C'est en effet habituel en sciences de gestion que de parler de « résistance au changement », en valorisant implicitement le point de vue des « décideurs » au détriment de celui des « exécutants ». Inversement, la sociologie du travail a particulièrement développé la thématique de la résistance, par exemple celle des ouvriers vis-à-vis de la maîtrise. Dans les deux cas, le clivage est hiérarchique. 
frustration de l'encadrement (qui voyait là une contrainte opérationnelle de plus à prendre en compte) et des agents présents sur le terrain. Pour la majorité des contrôleurs, la BA se faisait en effet au détriment du contrôle répressif. Dans les termes de la clinique de l'activité, cette situation était génératrice d'une forme d' «activité contrariée » (Clot, Faïta, Fernadez, $\&$ Scheller, 2000) puisque la BA venait (aux yeux des agents) contrarier les pratiques de contrôle traditionnelles (fondée sur la logique répressive) et bousculer le "genre professionnel » en vigueur.

Cette contrainte s'avérait d'autant plus difficile à accepter qu'elle ne relevait pas du contrôle répressif. La $\mathrm{BA}$ représentait en effet une avancée décisive des tenants de l'approche attentionnée du contrôle. Ainsi, pour l'observateur à la découverte du CSB, la BA fonctionnait comme un prisme qui mettait en lumière les lignes de rupture à l'intérieur du corps de contrôle : évoquer la BA avec les acteurs du CSB, c'était en fait bien souvent leur demander de prendre position. Nous avons déjà précisé les différences de «philosophie » du contrôle derrière ces deux organisations du travail. Il nous faut également souligner que ces différences ne suscitaient pas d'échanges ou de controverses sur le travail de contrôle susceptibles de faire évoluer le «genre professionnel » (Clot, 2001) existant, mais plutôt des ruptures.

\section{Des ruptures historiques ancrées dans le quotidien}

Ces ruptures contrôle répressif / attentionné étaient d'ailleurs déjà solidement installées au CSB. Le cas du SDL en est l'illustration très concrète. L'observateur à la rencontre du SDL, à mesure qu'il découvre l'histoire de sa constitution (par la présentation qui en est faite par le responsable, en discutant avec les agents de la première heure, au cours de l'entretien avec le responsable du contrôle traditionnel du secteur correspondant, etc.), se rend compte de la séparation qui s'est irrémédiablement mise en place.

Celle-ci se traduit tout d'abord dans la séparation des locaux : les ALGECO du SDL sont situés dans le même dépôt que les bâtiments du secteur 5 du CSB, mais ils en sont distants d'une cinquantaine de mètres et disposés de telle façon que les agents ne se croisent pas pour y accéder. ${ }^{17}$ En termes de mobilité, on peut noter également que les agents du SDL, pour ceux issus du contrôle ${ }^{18}$, étaient détachés de leur secteur; mais il n'était pas question d'un retour et le management des détachés (concernant l'évaluation par exemple) était complètement délégué au responsable du SDL.

Enfin, dans les discours des agents et de l'encadrement de proximité, d'un côté comme de l'autre, revenaient les dénonciations des valeurs comme des pratiques des autres. Par exemple, d'après les agents qui avaient choisi le SDL, il s'agissait de réfléchir plutôt que de verbaliser par réflexe ou par idéologie; alors que pour les tenants du contrôle répressif, l'approche attentionnée cachait un renoncement face à la difficulté. Il existait donc un véritable clivage.

Cependant, cette situation présentait un avantage indéniable dans la comparaison avec la BA : celui de préserver l'autonomie des tenants du contrôle attentionné, pour laisser le temps à des pratiques alternatives d'émerger et de se développer, voire à un «genre professionnel » alternatif d'éclore... D'autant plus que les équipes rattachées au SDL se composaient à la fois de contrôleurs détachés du CSB et de machinistes-receveurs, tous volontaires pour expérimenter de nouvelles pratiques professionnelles, voire pour contribuer à l'émergence d'un nouveau «métier » au sein de la RATP...

En comparaison avec la situation du SDL décrite ci-dessus, la BA avait l'inconvénient d'être perçue comme une menace par la majorité des contrôleurs du CSB, dans la mesure où elle s'inscrivait en décalage par rapport aux pratiques de métier considérées comme légitimes par le corps social du CSB. En outre, la BA était finalement ambiguë et aucun camp ne pouvait

17 Notons toutefois qu'une séparation géographique aussi nette n'était pas systématique, puisque les locaux du NST 2 se trouvaient à l'intérieur des locaux d'un secteur CSB classique.

18 Les machinistes-receveurs volontaires y contribuaient également, alternant entre la conduite et le contrôle. 
se l'approprier complètement: très éloignée du contrôle répressif, elle faisait figure de repoussoir pour la plupart des agents ; proche, dans son inspiration, du contrôle attentionné tel qu'il se donnait à voir au SDL ou au NST, elle n'en avait ni l'échelle ni les hommes ni la dynamique, puisque c'était l'ensemble du CSB qui la mettait en œuvre. En comparaison avec le SDL, il semblait plus difficile, sinon impossible, de faire émerger via la BA un « genre professionnel » alternatif, encore moins un nouveau «métier », d'autant plus que la BA est apparue, non seulement comme une contrainte, mais comme une coquille vide. Nous récapitulons ces éléments dans le tableau 1.

$\mathrm{Au}$ final, avec la mise en œuvre de la BA et à travers les oppositions fortes qu'elle a révélées, c'était une problématique de renouvellement du «genre professionnel », tel qu'il se donnait à voir au CSB, que l'observateur devait élucider, puisqu'aucune controverse ne venait revivifier le «genre du métier» de contrôleur.

\begin{tabular}{|c|c|c|c|}
\hline & «Philosophie » du contrôle & $\begin{array}{l}\text { Rattachement } \\
\text { institutionnel }\end{array}$ & «Dynamiques » à l'oeuvre \\
\hline $\begin{array}{l}\text { Contrôle } \\
\text { répressif }\end{array}$ & $\begin{array}{c}\text { De grandes équipes } \\
\text { intervenant ponctuellement } \\
\text { pour constater des infractions } \\
\text { et dresser des PV }\end{array}$ & $\begin{array}{l}\text { Les agents appartiennent } \\
\text { au CSB (rattachés à un } \\
\text { secteur donné), le contrôle } \\
\text { répressif y est très } \\
\text { largement majoritaire et } \\
\text { est valorisé }\end{array}$ & $\begin{array}{l}\text { Présence d'un « genre du } \\
\text { métier », fondé sur la } \\
\text { logique répressive, et rigide }\end{array}$ \\
\hline $\begin{array}{l}\text { Contrôle } \\
\text { attentionné }\end{array}$ & $\begin{array}{c}\text { De petites équipes présentes } \\
\text { sur la durée sur des lignes } \\
\text { spécifiques « travaillent » les } \\
\text { comportements des voyageurs } \\
\text { (validation par ex.) } \\
\text { principalement en amont } \\
\text { (fidélisation commerciale, } \\
\text { pédagogie des règles) }\end{array}$ & $\begin{array}{c}\text { Les agents sont détachés } \\
\text { de leur secteur CSB pour } \\
\text { participer aux } \\
\text { expérimentations (SDL, } \\
\text { NST) }\end{array}$ & $\begin{array}{c}\text { Émergence et } \\
\text { développement de pratiques } \\
\text { alternatives, voire d'un } \\
\text { «genre professionnel » } \\
\text { alternatif (néanmoins } \\
\text { fragile...) }\end{array}$ \\
\hline Bus Attitude & $\begin{array}{l}\text { Proche du contrôle } \\
\text { attentionné (petites équipes, } \\
\text { règles du savoir voyager) }\end{array}$ & $\begin{array}{l}\text { Les agents du CSB sont } \\
\text { réquisitionnés pour le } \\
\text { projet d'entreprise, à } \\
\text { hauteur d'environ } 50 \% \text { de } \\
\text { leur temps de travail }\end{array}$ & $\begin{array}{l}\text { Projet ambigu, mal compris } \\
\text { par les agents, vide de sens, } \\
\text { générateur d'une « activité } \\
\text { contrariée »(Clot et al., } \\
2000)\end{array}$ \\
\hline
\end{tabular}

Tableau 1 : Tableau récapitulatif des types de contrôle

Table 1: Table summarising the different control types

\section{3.- Les indicateurs de gestion comme facteur de blocage}

De nombreux facteurs expliquent cette situation, au nombre desquels on peut notamment ranger la faible mobilité des agents de contrôle au sein de la RATP. ${ }^{19}$ Mais l'observation a permis de mettre en évidence une autre raison à cet état du métier de contrôle à la RATP : les indicateurs de l'activité de contrôle. Au-delà des nombreux tâtonnements dans la mise en œuvre opérationnelle du projet (peut-on verbaliser dans le cadre de la BA ? Quand doit-on recourir au dispositif d'intervention prévu pour les voyageurs retors aux règles du "savoirvoyager»? etc.), c'est en effet notamment sur le plan du compte-rendu de son activité que la BA a «péché », si l'on se fie au point de vue des agents.

Non pas que cette question n'ait pas été anticipée par les pilotes du projet. Ne serait-ce que pour leur propre usage, ces derniers disposaient d'une batterie d'indicateurs pour suivre la production des agents. Ainsi, bien qu'ils puissent disparaître d'un document analysé à l'autre, on pouvait trouver pris en considération des indicateurs spécifiques comme le nombre de tickets vendus à bord du bus, au cours de la présence des équipes BA, ou encore l'évolution du nombre d'abonnements vendus, à côté des indicateurs les plus significatifs

19 En effet, les perspectives d'évolution pour les agents de contrôle étaient relativement limitées, notamment au moment de l'enquête, puisque le taux d'encadrement était déjà jugé trop élevé au CSB, et que le CSB avait mauvaise réputation en interne (aux dires des acteurs). Le marché interne de l'emploi n'était donc pas très favorable ; quant au marché externe, compte tenu des conditions d'emploi offertes par la RATP, nombre d'agents ne considéraient pas comme une option le fait de quitter la Régie. 
pour l'encadrement opérationnel et les agents de contrôle, à savoir le nombre de PV rédigés et le nombre de voyageurs contrôlés.

L'existence conceptuelle de ces indicateurs spécifiques ne dit rien cependant de la façon dont ils apparaissaient aux agents de contrôle, ou autrement dit, dans une approche discursive des indicateurs de gestion (Honoré, 2007), de ce qu'ils disaient aux agents sur la BA. En effet, ce dont rend compte le point de vue des agents, c'est que ces indicateurs ne leur apparaissaient pas toujours comme pertinents à témoigner de leur travail, soit parce qu'ils n'étaient pas repris dans les tableaux de bord (par exemple les abonnements dans le rapport d'activité d'un secteur), soit parce que leur hiérarchie directe ne reprenait pas les chiffres reportés bus après bus par les agents sur la «feuille de route » de la BA, soit enfin parce que les bilans de la BA, pourtant communiqués vis-à-vis de l'extérieur de la RATP ${ }^{20}$, n'étaient pas affichés dans les locaux où les agents prenaient leur service... Autant dire que cette situation a été préjudiciable à la $\mathrm{BA}$, puisque dans la comparaison avec le contrôle traditionnel ou le SDL (voir ci-dessous), aucun indicateur crédible n'est venu soutenir et légitimer la BA.

\section{Un point de comparaison : le management par les indicateurs au CSB et au SDL}

Ce manque de légitimité des indicateurs spécifiques à la BA contraste fortement avec l'attention qu'accordaient (et le temps que consacraient) les acteurs, agents comme responsables, aux indicateurs de production habituels. Au CSB, chacun prêtait attention à l'indicateur le plus proche de son niveau d'activité et le plus valorisé par son groupe d'appartenance. Les agents de contrôle se focalisaient sur le nombre d'affaires réalisées, quand les responsables d'équipe et de secteur veillaient également au respect du taux de contrôle minimum sur les différentes lignes. Ces indicateurs étaient très régulièrement évoqués, à l'intérieur de l'équipe, entre deux bus, mais aussi lors des entretiens d'évaluation et lors des briefings d'équipe.

La situation était très différente dans le cas du SDL puisque le responsable du service avait élaboré son propre tableau de bord et institutionnalisé des indicateurs alternatifs à même de rendre compte de l'activité de ses agents. Ces indicateurs étaient largement utilisés dans l'animation quotidienne de son équipe (suivi de la production, briefing du matin...) et dans la communication de ses résultats vis-àvis de sa hiérarchie. Pour rendre compte du travail réalisé, les agents remplissaient une feuille de route structurée à partir de ces indicateurs. Cette prise au sérieux des indicateurs de production correspondait à un objectif qui, pour le responsable du service, n'était pas que le pilotage de l'activité. En fait, à travers ces indicateurs alternatifs et la série de chiffres qu'ils permettraient d'obtenir à terme, il s'agissait de se donner des arguments pour légitimer le contrôle attentionné - en objectivant la comparaison avec le contrôle répressif. Cette prise au sérieux par l'encadrement allait de pair avec l'observation auprès des agents d'une prise au sérieux de l'activité elle-même et était de nature à légitimer les pratiques relevant du contrôle attentionné, voire à encourager l'éclosion d'un « genre professionnel » alternatif ${ }^{21} \ldots$ Il s'agit d'une différence notable avec la BA.

Les agents en BA subissaient-ils cette disparition des traces de leur activité, ou bien en étaient-ils les complices, prompts à saisir l'absence d'institutionnalisation des indicateurs spécifiques à la BA comme prétexte pour dénoncer la vacuité de la BA ? À vrai dire, le terrain n'a pas permis de trancher entre ces deux options. Mais là n'est pas l'important : il s'agit plutôt de souligner combien l'absence d'indicateurs visibles et pertinents pour les agents, dans la comparaison avec le contrôle répressif (voir l'encadré «La prégnance de la

20 Ainsi, l'observateur était confronté en permanence à un paradoxe : les chiffres de satisfaction des « clients » concernant la BA étaient tout à fait positifs, et la direction de BUS utilisait ce résultat comme argument pour justifier de la légitimité et de la pertinence du projet, alors que dans le même temps, sur le terrain, les équipes pensaient que «la BA ça ne marche pas ».

21 A noter que pour Yves Clot plusieurs « genres professionnels » peuvent se concurrencer au sein d'un même métier (2008). En effet, pour lui, «il n'y a pas d'harmonie rigoureuse et définitive entre le métier et le genre professionnel où il prend place dans l'activité. Plusieurs genres professionnels peuvent se recouper au sein du même métier et même s'y trouver en conflit.» (Clot, 2008, pp. 261-262). 
verbalisation dans les pratiques professionnelles»), a laissé la porte ouverte à ce commentaire si souvent entendu : «faire de la BA, c'est ne rien faire ».

\section{3.- Les indicateurs d'une gestion multimodale de la fraude}

À la suite de cette analyse des transformations de la lutte contre la fraude, le chercheur a proposé à la direction du CSB une représentation graphique, dénommée «schéma de l'escalier des modes d'action» (voir Annexe 1). Ce schéma reposait sur l'idée selon laquelle à chaque type de contrôle correspond une rationalité et une compréhension spécifique de la fraude $^{22}$. La direction du CSB trouva ce schéma très parlant et le directeur proposa d'associer le jeune chercheur à un groupe de réflexion sur le pilotage de la lutte contre la fraude. Le chercheur-intervenant apparaissait comme une « ressource » pour alimenter les réflexions du groupe, d'autant plus qu'on le créditait désormais de «compétences de conceptualisation » (Suquet, 2008). Cette remontée dans l'épaisseur de l'organisation était notamment l'occasion de recueillir des données sur les modes de production des indicateurs de gestion. La démarche a consisté, au fur et à mesure d'une série de réunions de fin août 2005 à avril 2006, à concevoir une organisation «multimodale» de la lutte contre la fraude, autrement dit articulant différents types de contrôle (et faisant entre autres une place à la Bus Attitude).

\section{1.- Vers un pilotage multimodal de la lutte contre la fraude}

Le directeur du CSB avait constitué un groupe de travail, à la croisée d'une démarche de certification ISO et d'une décision stratégique d'avancer vers un contrôle multimodal. Le groupe devait notamment travailler à l'opérationnalisation du processus dit de «planification des missions de contrôle». En l'occurrence, l'échéance la plus directe était celle de la création des outils permettant de mettre en œuvre ce processus au quotidien.

Le rôle du chercheur dans le groupe consistait à stimuler le groupe dans sa réflexion sur le pilotage de la lutte contre la fraude. Ce groupe était constitué par ailleurs de trois responsables opérationnels chevronnés et du directeur adjoint du CSB. Le directeur avait considéré que la présence récente du jeune chercheur dans l'entité lui donnait un regard neuf à même de bousculer certaines certitudes et habitudes des responsables, plus anciens ( « décoiffer » était son terme exact), et ce d'autant plus que le temps passé sur le terrain lui donnait une légitimité suffisante pour prendre place au sein du groupe et participer à ses discussions.

Le projet étant jugé stratégique et sensible, à aucun moment il n'a été question pour le directeur du CSB de faire participer des contrôleurs à la réflexion. Impliquer des responsables hiérarchiques ou des acteurs ayant une bonne connaissance du «terrain » et des différents modes de contrôle (ce qui était le cas des trois responsables opérationnels et du chercheur), sans pour autant solliciter des agents de «la base », était aux yeux du directeur du CSB une garantie pour éviter d'éventuels « dérapages $»^{23}$. Signalons également qu'aucun responsable opérationnel des NST 1 et 2, ou du SDL, ne fût désigné par le directeur pour participer à ces réunions. La présence de son adjoint et du jeune chercheur dans le groupe (deux acteurs qui connaissaient bien ces expérimentations) lui semblait amplement suffisante.

L'intervention du chercheur ne pouvait concerner l'ensemble des outils mis en place. Et il fut convenu avec l'animateur du groupe qu'il devait mobiliser les connaissances qu'il avait

22 A noter que l'élaboration de ce schéma n'aurait pas été possible sans au préalable cette immersion de longue durée au sein du CSB.

23 Il faut dire que le déploiement de la BA au sein du CSB s'était traduit par l'apparition de tensions importantes au sein des équipes et par une démotivation générale des agents. Dès lors, pour le directeur, la prudence était de mise. 
acquises suite à ses observations de terrain pour concevoir un outil qu'il présenterait comme une base de discussion au groupe : la «matrice des modes d'action $»^{24}$ (voir Annexe 2). Cette dernière était envisagée comme une description synthétique des différentes techniques de contrôle existant à la RATP (en l'occurrence, principalement le contrôle répressif, le contrôle attentionné, et la BA). Dans l'esprit de l'animateur et du chercheur, il s'agissait d'un outil permettant de confronter les modes d'action sur la fraude et de susciter des controverses de métier au niveau de l'encadrement opérationne ${ }^{25}$. Cette confrontation devait avoir lieu dans un premier temps au sein du groupe de travail, pour établir une version de référence de la matrice, avant que celle-ci ne prenne place dans un second temps comme un rouage du système de pilotage de la lutte contre la fraude multimodale.

Le groupe a tout d'abord accepté le principe même de la matrice et de son élaboration en commun. Il a ensuite validé au cours d'une seconde réunion les critères d'évaluation des différentes techniques de contrôle retenus par le chercheur (et les indicateurs qui les soustendaient). Signalons que sans une connaissance fine du terrain et des différents modes de contrôle, le chercheur n'aurait pas été en mesure de proposer des critères et des indicateurs d'évaluation des différentes techniques de contrôle. L'analyse préalable de l'activité des contrôleurs et des encadrants a ainsi offert au chercheur des points d'appui pour concevoir des critères et des indicateurs de gestion. Les réunions suivantes ont été consacrées au remplissage de la matrice, c'est-à-dire à l'évaluation concrète des différentes techniques de contrôle.

Cette évaluation se focalisait sur deux aspects principaux de la description : l'instrumental et l'économique. Concernant l'aspect instrumental, il renvoyait à la caractérisation de l'agencement organisationnel et de la technique de contrôle : taille de l'équipe, connaissance requise de l'environnement (secteur ou ligne), recours à une assistance extérieure, type de production (PV, ticket vendu, abonnement...). L'aspect économique envisageait le périmètre du réseau couvert par une équipe recourant à une technique de contrôle, le nombre de voyageurs rencontrés, le nombre d'accidents du travail, la finesse de la «maille du filet » (cette technique laisse-t-elle passer de nombreux fraudeurs ?) et l'efficience de l'action.

Si le principe de la matrice avait été accepté sans réticence («ça nous fait beaucoup avancer », disaient-ils en chœur), de nouveaux échanges ont pourtant été nécessaires pour se mettre d'accord sur les caractéristiques de comparaison des modes d'action à retenir, et sur la façon de remplir chacune des colonnes ${ }^{26}$.

L'élaboration des caractéristiques de la matrice s'est donc faite par itérations, les responsables opérationnels y participant grandement. Ainsi, à titre d'exemple, pour évaluer chaque type de contrôle quant à la connaissance de la cartographie, les membres du groupe ont dû préciser à la fois ce que comprenait la cartographie et son extension géographique. «Connaître sa cartographie », était-ce savoir situer les arrêts du bus ? Les correspondances ? Les bâtiments publics importants? Les distributeurs automatiques? Et d'autre part, afin de comparer la qualité des connaissances des techniques de contrôle, il fallait trouver une extension commune : la ligne fut finalement choisie comme unité de comparaison (plutôt que le secteur).

24 Il s'agissait en quelque sorte d'opérationnaliser le « schéma de l'escalier des modes d'action » en vue de la mise en place d'une organisation multimodale de la fraude.

25 Dans la mesure où sur le terrain la mise en place de la BA n'avait pas suscité de véritables controverses de métier parmi les contrôleurs, mais plutôt des ruptures, comme l'a révélé l'immersion du chercheur au sein du $\mathrm{CSB}$, la mise en place de ce groupe de réflexion était l'occasion de susciter des controverses de métier au niveau de l'encadrement, en limitant les risques induits par cet exercice puisqu'il s'agissait, pour le directeur du CSB, de mener une réflexion «en chambre ».

26 Ajoutons que les termes proposés par le chercheur, et explicités à partir d'un glossaire, n'ont pas toujours été immédiatement clairement définis ou compris ; ils ont souvent fait l'objet d'une redéfinition de la part des praticiens (« je traduis par... »). 
Nous présentons une version intermédiaire de la matrice (voir Annexe 2). Bien que chaque réunion ait permis d'avancer vers une version aboutie de la matrice, cette dernière n'a jamais vu le jour, ce qui révèle rétrospectivement sa véritable utilité : la matrice servait d'aiguillon pour les discussions du groupe, auxquelles elle offrait un support en même temps qu'un horizon. Discuter à travers la matrice sur les indicateurs pour évaluer les techniques de contrôle a ainsi donné l'opportunité d'un dialogue sur le contrôle, dont nous allons rendre compte maintenant.

\section{2.- L'opportunité de faire dialoguer certains acteurs et de stimuler une controverse sur le contrôle}

Les réunions du groupe n'ont la plupart du temps pas abouti à des chiffres exacts. Cependant, elles ont toujours donné l'occasion de comparer les techniques de contrôle pour les hiérarchiser. La hiérarchisation était d'ailleurs prétexte à l'affrontement des techniques de contrôle les unes avec les autres. Cet affrontement a permis de mettre en lumière les hypothèses des acteurs de la lutte contre la fraude en présence, d'une part sur un plan moral, et d'autre part sur un plan instrumental.

En effet, cherchant à comparer l'apport des différents types de contrôle, les participants du groupe de travail ont échangé des propos assez vifs au sujet de la BA. Pour les opposants à celle-ci (les trois responsables opérationnels chevronnés), quand ce n'était pas « de la m...», c'était «un boulot de fainéant». Pour reprendre une analogie plusieurs fois exprimée par l'un des responsables opérationnels, la mise en place de nombreux radars sur les autoroutes montre bien que ce qui est efficace, c'est la répression, et que « la BA ne sert à rien »...

À l'appui de ce constat, mentionnons que le chercheur a lui aussi dû prendre position, contraint par la réaction et les interpellations des autres responsables opérationnels. Sans qu'il ne se soit jamais senti un partisan de la BA, il en vint à la défendre face à la virulence des responsables opérationnels, qui lui semblait souvent exagérée. Sa connaissance fine du contrôle attentionné et des principes sous-tendant la BA lui a ainsi permis de souligner les avantages des techniques de contrôle alternatives par rapport au contrôle répressif. L'élaboration d'un outil commun s'est ainsi faite par la répartition du groupe de travail en deux camps: les partisans (l'animateur et le chercheur) et les opposants à la BA (les responsables opérationnels).

Une telle répartition du groupe en deux camps est à notre sens notable, parce qu'il ne s'agit pas d'une séparation, comme c'était le cas dans les secteurs (voir ci-dessus). Au contraire, et aussi tendu qu'ait été parfois le ton des échanges au cours des réunions, celles-ci n'ont jamais cessé d'être le cadre d'un dialogue et d'une dynamique d'argumentation (parfois à court d'arguments toutefois...). Elles ont néanmoins permis de révéler les lignes de tension du « réel de l'activité $»^{27}$ (Clot et al., 2000), quand bien même le terrain était éloigné.

Il est frappant d'ailleurs de remarquer combien les lignes de tension apparues au cours des réunions rejoignaient celles que le chercheur avait pu observer directement au contact du «terrain». Cela s'explique par le profil des autres membres du groupe, responsables opérationnels eux-mêmes très proches du terrain et conscients du «réel de l'activité » des agents de contrôle, puisqu'ils en organisaient le travail quotidien.

En définitive, ce que révélaient ces réunions sur un plan moral c'est non seulement la prégnance des pratiques de contrôle habituelles (basées sur l'interpellation des fraudeurs et la sanction), mais ce sont aussi les refus d'une évolution du « genre du métier » de contrôleur.

27 Le « réel de l'activité », selon la clinique de l'activité, est « ce qui ne se fait pas, ce qu'on cherche à faire sans y parvenir (...), ce qu'on aurait voulu ou pu faire, ce qu'on pense pouvoir faire ailleurs, mais aussi paradoxalement ce qu'on fait pour ne pas faire ce qui est à faire, ou encore ce qu'on fait sans vouloir le faire » (Clot \& Litim, 2003, p. 1544). 
Sur un plan instrumental, la remise en cause de la BA avait ceci d'intéressant qu'elle passait souvent par une controverse sur la possibilité d'évaluer la production de la BA, et donc sur la légitimité de tel ou tel indicateur pour évaluer l'activité des agents et la production correspondante. Certaines controverses récurrentes n'ont pas été surmontées et ont, au final, empêché l'élaboration d'un nouvel indicateur (qui aurait permis de légitimer la BA).

Ainsi, au cours d'une réunion, réfléchissant aux indicateurs pertinents pour le pilotage envisagé, le coordinateur du groupe, pourtant lui-même favorable à la BA, interrogeait : «ça fait bien quelque chose ?»; mais lorsque le chercheur suggéra de comptabiliser les tickets vendus dans le bus en la présence des équipes en ligne (prenant exemple sur les pratiques du SDL), celui-ci fut plus dubitatif : " c'est de l'incitation, concrètement, ils ne font rien ».

Il rejoignait alors le sentiment général des autres membres du groupe, qui considéraient qu'il y avait avant tout production d'un «sentiment de sécurité pour les clients qui payent », mais que « ce n'est pas mesurable ». Et en conséquence de cette prise de position partagée, il n'y a pas eu d'indicateur pour rendre compte de cet aspect de l'action des agents en BA. Ceci est d'autant plus frappant peut-être que le même type de critique pouvait être adressé à l'efficacité de la verbalisation (quel lien y a-t-il entre celle-ci et l'évolution du taux de fraude ? Et comment le mesurer ?), mais que les responsables opérationnels ne l'entendaient pas.

Le résultat des réunions n'a ainsi pas toujours été à la hauteur des espérances initiales du chercheur, notamment en termes de production d'indicateurs nouveaux. Mais quel qu'ait été au final la capacité du groupe à inscrire dans la matrice le résultat des discussions, il faut mentionner une plus grande tolérance à l'égard de la BA, dont témoigne d'ailleurs bien l'assertion interrogative de l'animateur du groupe : «ça fait bien quelque chose? ». En acceptant de considérer la BA au regard du contrôle traditionnel, les responsables opérationnels ont pu faire évoluer, sur certains points et dans une certaine mesure, leur conception de la lutte contre la fraude.

Une illustration en est le débat à propos du taux de contrôle. Les responsables opérationnels trouvaient injuste que soient mis sur le même plan le taux de contrôle du contrôle répressif et celui du contrôle attentionné et de la BA: seul le contrôle répressif était vraiment du contrôle. Ceci s'expliquait par le fait qu'il n'y avait qu'un seul indicateur existant, ce qui générait l'amalgame des productions. La discussion au sein du groupe a permis de mettre en évidence le besoin d'un nouvel indicateur qui rende visible ce qu'il y avait de spécifique et de valorisable dans le «contrôle » de la BA et du SDL / NST. Cela a été l'occasion pour les membres du groupe d'échanger sur l'activité des agents et sur l'intérêt des modes de contrôle alternatifs (Comment les agents se positionnent-ils lorsqu'ils sont en BA ? Quelle ambiance la BA produit-elle ? Quelles sont les difficultés rencontrées ? etc.) pour construire un nouvel indicateur. Ainsi, on a alors parlé de « voyageurs rencontrés» et de "probabilité de contact pour un voyageur», ce qui a permis de distinguer les modes d'action. Les responsables opérationnels ont alors accepté de reconnaître l'intérêt des modes d'actions « incitatifs » (BA et SDL / NST) par rapport à l'approche répressive.

Là encore, bien que ne s'étant pas traduit concrètement par une évolution en conséquence de la matrice (les cases «nombre de voyageurs rencontrés » sont restées remplies de points d'interrogation...), il a permis un échange d'arguments. Ainsi, par le truchement de la remise en cause de la BA comme activité de lutte anti-fraude, des indicateurs de son activité, le groupe en est venu à interroger l'activité de la BA elle-même, d'une part, mais aussi, puisque c'est inséparable, l'activité de contrôle traditionnel. Il faut souligner, néanmoins, les points aveugles de cette interrogation, et les difficultés réelles à statuer sur les résultats en la matière.

\section{3.- Les difficultés et les résultats mitigés}

En effet, un tel processus d'apprentissage n'a de sens que s'il est remis en perspective avec 
les transformations de l'activité qui s'en suivent éventuellement. Or de ce point de vue, les résultats sont apparus limités. Nous avons déjà souligné en effet combien les présupposés étaient parfois résistants, fondés sur des convictions solides (ce dont témoigne la comparaison avec les radars sur les routes), qui ne se sont pas autant estompées que l'aurait souhaité l'intervenant. Les indicateurs nouveaux censés rendre compte plus justement de ce que produisait la BA n'ont pas réellement vu le jour, comme nous l'avons mentionné.

D'autre part, et bien que le groupe soit parvenu à une organisation du contrôle multimodal, i.e. donnant une place à la fois à la $\mathrm{BA}$ et au contrôle répressif sur une même ligne sur une séquence de quelques semaines, il est notable que la place réservée à la première dans les «scénarii de contrôle » la réduisait à un pré-contrôle (certains participants ont utilisé la métaphore des macarons verts placés comme avertisseurs sur les pare-brises des voitures mal stationnées) ou à une activité indifférenciée («on met un peu de BA»). Les scénarii étaient très clairement organisés autour du contrôle répressif, qui restait premier dans l'esprit des responsables opérationnels. En définitive, d'un point de vue organisationnel, la BA ne pouvait pas réellement faire évoluer le «genre du métier» de contrôleur, dans la mesure où les acteurs n'en rendaient compte qu'en marge de l'activité (le contrôle répressif restant le cœur du métier).

Dernier point, qui amène l'intervenant à beaucoup de prudence sur les effets réels de l'intervention (au-delà du fait qu'il n'a pas été possible de retourner sur le terrain juger des effets concrets sur l'activité des agents du CSB), la matrice est rapidement tombée dans l'oubli... Curieux paradoxe, qui veut que les participants du groupe aient vraiment pris le temps d'échanger au cours des réunions, qui duraient chacune plusieurs heures, mais ne se souviennent plus vraiment de ce dont il s'agissait une fois quelques mois passés, lorsque l'intervenant les interroge sur le devenir de la réflexion commune. Ce paradoxe demande de revenir sur les conditions de cette intervention pour envisager les conditions d'une intervention « réussie » par les indicateurs sur le travail.

\section{4.- Quels principes d'intervention en déduire ?}

\section{1.- Intérêts et limites d'une intervention sur le travail par les indicateurs de gestion}

Il nous semble ressortir tout d'abord de l'analyse de cette intervention l'intérêt d'une approche de l'activité par les indicateurs de gestion. En effet, l'analyse des indicateurs de la lutte contre la fraude est venue compléter l'analyse de l'activité des agents de contrôle et de l'encadrement de proximité réalisée lors de la phase d'immersion du chercheur au sein du $\mathrm{CSB}$ et a permis de confirmer un certain nombre d'hypothèses (élaborées lors de la première phase de la recherche) : - les focalisations des acteurs (sur le procès-verbal par exemple) ; leurs façons de percevoir leur environnement (des clients potentiels à fidéliser et à éduquer pour les tenants du contrôle attentionné, des fraudeurs éventuels à prendre sur le fait pour la majorité des agents, qui considèrent que le seul «vrai » contrôle est réalisé en chute); - des façons de donner du sens au travail... C'est alors l'absence de visibilité des indicateurs de la BA que l'on peut pointer, par comparaison avec le tableau de bord de proximité mis en place au SDL, mais aussi par comparaison avec un certain nombre d'indicateurs "prégnants » (Boussard, 2001) - par exemple le nombre de PV réalisés - qui ont pour effet de valoriser le contrôle répressif et de décrédibiliser, par la même, les pratiques alternatives.

Ces focalisations, perceptions de l'environnement et façons de donner du sens au travail, prises ensemble, donnent à voir une dynamique plus générale du travail de contrôle à la RATP : celle d'un «genre professionnel » qui a le plus grand mal à se renouveler et qui se signale plus par les frontières étanches entre les différentes lignes du contrôle, que par la vitalité des disputes professionnelles au sein du service. Cela nous semble d'autant plus probant et intéressant que cette mise en évidence ne s'est pas faite uniquement au niveau des 
opérateurs ; bien plus, les indicateurs nous ont permis de « remonter » jusqu'aux encadrants, et de montrer leur contribution à cette dynamique. En d'autres termes, les indicateurs dessinent les contours du «genre professionnel» dans sa globalité ; ils soulignent la continuité entre des niveaux hiérarchiques, trop souvent isolés, voire opposés dans l'analyse du travail.

Pour autant, le récit traduit de façon criante un certain nombre de limites dans les résultats de l'intervention réalisée à la RATP. Il y a tout d'abord un constat simple à faire : au moment où se termine le récit, autrement dit lorsque s'achève la présence du chercheur dans le service, on ne sait pas comment les indicateurs vont être repris sur le terrain : les nouveaux indicateurs vont-ils avoir un effet sur le travail de contrôle ? Et en effet, il y a là une double difficulté. D'une part, celle de l'horizon temporel de la recherche, et du temps qui lui est donné : suivons-nous des évolutions sur plusieurs années ou sur quelques mois ? D'autre part, une telle cécité témoigne d'une déconnexion par rapport au niveau des opérateurs, au «terrain ». Quand bien même on ne parle que de cela dans le groupe de travail, les membres qui le composent, et notamment le chercheur, en sont bien éloignés. Comment juger dès lors de la pertinence de la réflexion et de la réalisation collectives si on ne peut la ramener et la mesurer à l'aune de ce qui légitime la démarche d'intervention : le «terrain » ?

Il faut rappeler, enfin, que les indicateurs peuvent être de bien mauvais alliés pour transformer le travail. Comme la sociologie de la gestion l'a montré, certains seulement d'entre eux sont «prégnants » (Boussard, 2001) - et les autres ne prêtent pas vraiment à conséquence. D'ailleurs, quand bien même ils seraient également pertinents pour rendre compte du travail de contrôle, la concurrence installée entre les modes de contrôle avait pour corollaire un affrontement des indicateurs, dont les mieux installés sortaient le plus souvent vainqueurs. On a pu observer ce phénomène à l'intérieur du groupe de travail: les indicateurs du contrôle attentionné tels que le taux d'abonnement ou le nombre de tickets vendus, bien que connus des membres du groupe, n'étaient pas tant réfutés que rejetés derrière ceux du contrôle répressif dans la hiérarchie de l'attention; et au niveau du terrain le tableau de bord du SDL ne dépassait pas l'audience des membres du SDL et de quelques initiés.

Quand bien même les indicateurs étaient manifestement «prégnants » et revenaient souvent dans les débats, ce n'est pas pour autant qu'ils permettaient de poser les «bonnes» questions. On peut penser à ce sujet à l'absence de mise en doute de l'efficacité du PV, notée avec surprise par le chercheur. Il y avait là un véritable impensé collectif des encadrants, probablement très fondateur d'ailleurs de l'activité de contrôle, et demandant à ce titre à être investigué... Une telle découverte, qui ne peut être travaillée sur le vif avec les participants au groupe de travail, n'aurait-elle pas demandé une phase de restitution ultérieure, postérieure au travail sur les indicateurs?

\section{2.- Quels prolongements à cette expérience d'intervention ?}

Pour surmonter les difficultés d'une intervention par les indicateurs, nous proposons d'approfondir la réflexion méthodologique, d'une part en suggérant deux pistes de prolongement de l'intervention; d'autre part en revenant sur les enjeux principaux de la construction du rôle d'intervenant.

On l'a vu, l'élaboration de nouveaux indicateurs de la fraude à la RATP a tourné court. Elle n'a permis ni de poursuivre l'intervention pour constater les transformations du travail induites par les nouveaux indicateurs, et apprendre de celles-ci ; ni de continuer à travailler avec les encadrants membres du groupe de travail quant à leur propre compréhension des indicateurs et de l'activité de contrôle plus généralement. Il nous semble qu'il y a là deux directions possibles et opportunes pour prolonger l'intervention.

D'un côté, il s'agit d'anticiper, dès le départ de la recherche-intervention, l'importance de cette phase, que l'on pourrait qualifier de «bouclage». Dans notre cas, il y a bien eu un 
premier mouvement du «terrain» vers l'encadrement, mais il n'a pas été possible de retourner ensuite sur le «terrain». Pour être en mesure de bien travailler ce lien entre le travail à transformer et les indicateurs qui rendent compte de celui-ci, peut-être vaut-il mieux trouver une place plus proche de l'encadrement intermédiaire. Ainsi, on aurait pu envisager de participer à l'élaboration des indicateurs du SDL : les tableaux de bord «bricolés » par le responsable de cette petite équipe garantissaient une mise en regard directe du travail des agents et des indicateurs : comment sont-ils alimentés? En quoi semblent-ils orienter l'action ? Quels discours et pratiques soutiennent-ils ? Où et comment sont-ils affichés ? En quoi peuvent-ils contribuer à l'émergence et au développement d'un «genre professionnel » alternatif, voire d'un nouveau "métier»? etc. La situation du SDL aurait été d'autant plus intéressante à étudier que le cadre organisationnel et managérial propre au SDL semble être de nature à préserver les pratiques innovantes. Il s'agit d'une différence notable avec la BA. Du coup, on aurait également pu tirer les leçons de l'expérience du SDL pour positionner autrement la BA dans l'organisation en vue de soutenir le développement de pratiques de contrôle innovantes et de favoriser l'émergence d'un « genre professionnel » alternatif...

D'un autre côté, on peut discerner une autre piste prometteuse, pour poursuivre l'intervention : considérer les indicateurs comme une voie d'accès privilégiée à l'activité de l'encadrement. Alors que le travail sur les indicateurs a révélé les impensés et les façons de raisonner des membres du groupe de travail, il aurait été intéressant de compléter ce travail sur les indicateurs d'une restitution après coup à ce même groupe. La restitution aurait été l'occasion d'une mise en débat des observations et des analyses du chercheur, quittant pour le coup son statut de participant.

Bien entendu, cela revient à renoncer à une approche globale du «genre professionnel», pour se centrer sur la catégorie des encadrants. Mais il y a là assurément une piste d'autant plus intéressante que peu suivie en clinique de l'activité. Et puis, cette focalisation sur le travail d'encadrement ne nous semble pas amener mécaniquement une coupure d'avec le travail à transformer (celui des agents de contrôle). Les encadrants sont au contraire partie prenante de ce travail : dans notre cas ils étaient d'ailleurs les premiers intéressés par les retours «terrain» - retours qui constituaient autant d'occasions d'échanger sur un travail dont ils avaient une connaissance pointue, fondée notamment sur les retours quotidiens des agents de leur secteur.

Il ressort en tout cas de ces deux pistes des préoccupations méthodologiques : comment trouver la juste place d'intervenant pour agir sur le travail au moyen des indicateurs? Il est certain que la co-construction des indicateurs de gestion, si on la souhaite en tension avec le travail, ne peut procéder que d'une connaissance fine de l'activité et du rôle qu'y occupent les indicateurs. Il en va de la possibilité pour le chercheur de se faire reconnaître comme légitime pour parler du travail. En effet, puisqu'il s'agit de se situer à un niveau de la hiérarchie qui assure une connexion au terrain, il faut être capable d'échanger avec les responsables opérationnels, sur la base d'une expertise «comparable» du travail ${ }^{28}$. Or nous l'avons vu, ce qui caractérisait les membres de notre groupe de travail (principalement les trois responsables opérationnels chevronnés) était une connaissance fine du terrain - réduite au contrôle répressif certes, et donc parcellaire, mais fine néanmoins.

Cette acquisition d'une connaissance du travail peut se faire de plusieurs façons. Il semble possible d'en passer par un apprentissage à l'intérieur de l'organisation ou bien à l'extérieur pour partie. Ainsi dans le cas de notre recherche, le chercheur avait intégré la RATP après

28 Cependant, l'analyse de l'activité réalisée par le chercheur-intervenant, quand elle prend appui sur des méthodes éprouvées (ex : observation directe ou participante, auto-confrontations simples ou croisées, etc.), est susceptible de « révéler » des dimensions de l'activité qui n'apparaissent pas spontanément aux yeux des travailleurs eux-mêmes et de leur encadrement, et qui sont de ce fait difficiles à verbaliser et à expliciter (ex : les ficelles du métier, les connaissances et les compétences tacites, les routines et les «allants de soi » qui sont au fondement du métier, etc.). 
avoir déjà réalisé une recherche sur la fraude et le contrôle dans une autre entreprise de transport (Suquet, 2003). Cette première phase, comprenant de l'observation directe comme de l'observation participante du travail de contrôle, avait permis d'acquérir une certaine connaissance, y compris par corps, du métier, laquelle avait permis à son arrivée à la RATP de faciliter l'acceptation et l'observation sur le terrain de la RATP. Bien entendu il est incontournable d'en passer néanmoins par une phase d'imprégnation du contexte précis dans lequel se trouvent les acteurs de l'organisation étudiée.

Par ailleurs, en aval de cette première étape, il convient de réfléchir à la construction d'une place d'intervenant bien adaptée aux finalités de la recherche. Il y a en l'occurrence des dilemmes à arbitrer, dans la constitution du collectif de réflexion sur les indicateurs, d'une part, quant au rôle et à la posture critique du chercheur au sein du groupe, d'autre part.

Comme on a pu le voir, la composition du groupe de travail est décisive. On peut penser tout d'abord à la composition « horizontale » du groupe : à niveau hiérarchique équivalent, est-on en mesure de trouver dans le groupe des représentants de différentes activités, ou des tenants d'approches distinctes, voire rivales, d'une même activité ? La richesse des échanges qui en découlera est en jeu, ainsi que le succès de l'intervention, comme l'illustre bien le cas présenté, pour lequel le groupe de travail, rassemblant principalement des représentants du contrôle répressif, n'a pas pu aller au-delà d'un certain degré de remise en cause des fondements de cette approche de la lutte contre la fraude.

Mais la composition «verticale» du groupe est aussi importante. Le chercheur doit prêter attention au niveau hiérarchique auquel va se situer la réflexion collective. En effet, celui-ci est un bon indicateur de la proximité au terrain. Nous avons pu constater l'importance de maintenir une connexion au terrain pour le cas où le chercheur pense important d'accompagner les transformations du travail dans une phase supplémentaire de l'intervention. Ainsi, il s'agit de trouver un équilibre pertinent entre le terrain et les instances décisionnaires, à mi-hauteur de l'organisation, en travaillant avec des encadrants qui connaissent le «terrain » sans se confondre avec celui-ci.

\section{Le cas du CSB}

Dans le cas du CSB, dans la mesure où l'intervention avait révélé la prégnance de la verbalisation dans le maintien du «genre du métier» de contrôleur et dans les pratiques professionnelles, il aurait été particulièrement intéressant, dans la deuxième phase de l'intervention, de travailler avec d'autres encadrants de proximité, encore plus proches du terrain puisque responsables directs d'une équipe de contrôle, et pas seulement avec les responsables de secteurs ${ }^{29}$, pour co-construire des indicateurs de gestion alternatifs, et pour envisager le déploiement de ces indicateurs dans l'activité des agents, de façon à légitimer des pratiques alternatives encore fragiles et faire évoluer le « genre professionnel ».

En fonction de la composition du groupe de travail et de ses objectifs, le chercheur va être amené à se positionner de façon plus ou moins critique par rapport aux jugements des encadrants. La question est ici de savoir s'il est préférable pour le chercheur de suivre les réflexions entendues et de leur donner le statut de propositions pour l'action, valables en tant que telles; ou bien de remonter à partir de ces réflexions aux hypothèses qui les fondent, et de travailler sur ces hypothèses mêmes.

Par ailleurs, ce premier questionnement méthodologique amène à un autre questionnement : faut-il aller systématiquement à l'encontre des interprétations des acteurs ? Il peut s'agir d'une posture définie ex ante et assumée comme telle (bousculer les certitudes des acteurs et instaurer une dispute professionnelle) ou bien d'une adaptation pragmatique à une situation

29 Le CSB comptant à l'époque 800 agents environ, un secteur rassemblait en moyenne une centaine de contrôleurs. Les responsables opérationnels participant au groupe de travail géraient un secteur, et donc environ cent contrôleurs, ce qui ne leur permettait bien entendu pas la même proximité au terrain que celle des responsables d'équipes (entre 3 et 15 agents selon les lignes et les modes de contrôle décidés). 
déséquilibrée, comme dans notre cas où le chercheur s'est retrouvé, sans le souhaiter réellement, dans le rôle du défenseur du contrôle attentionné, tout simplement parce qu'il était quasiment seul à ne pas prôner systématiquement la verbalisation. Le risque pour le chercheur d'une posture peu consensuelle tient à sa possible exclusion de la réalisation collective et à sa non-association à la mise en œuvre des indicateurs sur le terrain...

Il est certain que ces questions ne sont pas anodines et méritent un examen attentif de la part d'un chercheur qui souhaiterait construire, avec des encadrants, les indicateurs permettant par cette démarche de transformer le travail. Clairement, les risques d'enfermement dans un schéma de pensée, de décision sans lendemain, ou encore de réflexion en salle sans connexion avec le terrain, sont importants ${ }^{30}$.

Enfin, rappelons le terme employé par les membres du groupe pour évoquer la participation du chercheur, dans notre cas : la «philosophie $»^{31}$. Terme prometteur s'il en est, tant la démarche qu'il annonce semble un antidote à la dévitalisation du «genre professionnel»; mais en même temps particulièrement dangereux : le philosophe et l'homme d'action ne font pas forcément bon ménage...

\section{Conclusion}

Nous avons montré, dans cet article, qu'il était difficile d'intervenir de façon efficace sur les indicateurs de contrôle en vue de transformer les pratiques professionnelles des agents de « la base » (ici les contrôleurs du réseau de surface de la RATP). D'une part parce que tel qu'il était structuré, le projet managérial qui servait de support à cette transformation (la démarche Bus Attitude) a été vivement critiqué par les agents et par l'encadrement opérationnel, mais aussi d'une certaine manière «détourné » : la réflexion sur la mise en place d'un contrôle multimodal de la lutte contre la fraude n'a pas abouti à une remise en cause des pratiques de contrôle habituelles et a contribué à la dilution de la Bus Attitude (celle-ci a été perçue au mieux comme un pré-contrôle, au pire comme une activité indifférenciée, donc vide de sens...). Les conditions d'une transformation des pratiques par les indicateurs n'étaient pas réunies dans ce contexte. D'autre part parce que la mise en œuvre de nouveaux indicateurs de gestion - en vue de soutenir de nouvelles pratiques de contrôle, plus pédagogiques et moins répressives... - s'est heurtée à un ensemble d'indicateurs «prégnants » (Boussard, 2001) qui légitimaient le contrôle répressif, sans que ces derniers soient réellement mis en discussion collectivement par les acteurs.

Ces indicateurs «prégnants » contribuent, de notre point de vue, au maintien du «genre du métier» (Clot \& Faïta, 2000) de contrôleur, au risque de le dévitaliser. C'est alors le développement du métier qui est entravé, «contrarié» (Kostulski et al., 2011). Par conséquent, il convenait de focaliser l'intervention "là où le développement du métier se trouve réellement «empêché » (Kostulski et al., 2011, p. 142), c'est-à-dire au niveau des indicateurs de gestion.

De ce point de vue, les résultats de la recherche-intervention sont contrastés. D'un côté, le suivi et l'analyse de la mise en œuvre de la Bus Attitude à la RATP, et la participation active du chercheur à la mise en place d'une organisation multimodale de la lutte contre la fraude (articulant différents types de contrôle), lui a permis de produire des connaissances sur le métier de contrôleur (identification du cœur du métier, via la mise à jour du «genre professionnel » qui le caractérise) en le «bousculant» et en questionnant le bien-fondé de l'ensemble des pratiques (du contrôle répressif au contrôle attentionné... en passant par la

30 D'où l'importance de la phase de bouclage avec le «terrain » mentionnée plus haut, bouclage qui n'a pas pu être opéré dans le cadre de la présente recherche.

31 L'animateur du groupe a ainsi lancé au chercheur, au cours d'une des réunions : «ça va c'est conforme à la philosophie ?». 
Bus Attitude) qui le constituaient à un moment donné. D'un autre côté, le chercheur a pu observer et analyser les forces de rappel à l'œuvre dans l'entreprise étudiée, forces incarnées à la fois par des acteurs (les agents de la base et l'encadrement opérationnel) et par des indicateurs de gestion solidement arrimés à l'organisation. Cette recherche-intervention a donc montré que l'évolution du «genre du métier» de contrôleur nécessitait une transformation des indicateurs de gestion pour soutenir des pratiques de contrôle innovantes et revivifier le «genre professionnel ». Mais la mise en place d'indicateurs alternatifs dans le cadre de la Bus Attitude s'est révélée particulièrement délicate, voire impossible durant le temps de la recherche-intervention, ce qui nous a conduits à proposer quelques pistes de réflexion, notamment sur un plan méthodologique.

Est-ce à dire pour autant que cette recherche-intervention s'est soldée par un échec ? Tout dépend à notre sens de la focale temporelle retenue. Durant le temps de la recherche, il n'a pas été réellement possible de transformer les pratiques de contrôle (sinon à la marge) et d'ouvrir le champ des possibles. Néanmoins, la Bus Attitude a permis aux acteurs de l'organisation (contrôleurs et encadrement opérationnel) de tester à grande échelle d'autres pratiques et de prolonger ainsi les expérimentations qui avaient été lancées dans le cadre des Nouveaux Services Tram 1 et 2 (NST 1 et NST 2) et du Service De Ligne (SDL). Un pas important a donc été franchi. Reste à franchir, selon nous, un second pas (plus difficile) : celui qui consiste à passer d'un «apprentissage en simple boucle» à un «apprentissage en double boucle » (Argyris \& Schön, 1978). Dans la mesure où la recherche-intervention a identifié un certain nombre d'impensés et d'angles morts ${ }^{32}$, et par effet de miroir révélé des croyances fortes partagées par le corps social du CSB, il nous semble effectivement nécessaire de travailler, avec les acteurs de l'organisation (agents de la base, encadrement opérationnel et dirigeants), sur les « conventions » qui soutiennent à la fois les indicateurs de gestion et les pratiques professionnelles. L'enjeu consiste, en définitive, à accompagner sur la durée les acteurs dans un questionnement sur les valeurs directrices et les croyances qui guident leurs représentations et leurs stratégies d'action.

\section{BIBLIOGRAPHIE}

Argyris, C., \& Schön, D. (1978). Organizational Learning: A theory of action perspective. Reading MA: Addison-Wesley.

Berry, M. (1983). Une technologie invisible ? L'impact des instruments de gestion sur l'évolution des systèmes humains. Paris: Centre de recherche en gestion de l'École Polytechnique.

Berry, M. (1986). Connaissance et action : de la balistique à la maïeutique. Communication au Colloque international d'HEC Montréal, 9-13 juin.

Berry, M. (1995). Research and the Practice of Management, a French View. Organization Science, 6(1), 104-116.

Bloomfield, B.P., \& Vurdubakis, T. (1997). Visions of organization and organizations of vision: The representational practices of information systems development. Accounting, Organizations and Society, 22(7), 639-668.

Boigne, J-M., Moisdon, J-C., \& Tonneau, D. (1986). Gérer ou comprendre ? Perplexités à propos d'une intervention en milieu hospitalier. Gérer et Comprendre, 5, 78-86.

Boigne, J-M., Moisdon, J-C., \& Tonneau, D. (1987). Gérer ou comprendre ? Perplexités à propos d'une intervention en milieu hospitalier. Gérer et Comprendre, 6, 72-81.

Boussard, V. (1999). Construction et objectivation de la légitimité des règles : le cas des indicateurs de gestion. Paris: Thèse de doctorat de l'Université de Paris IX.

Boussard, V. (2001). Quand les règles s'incarnent. L'exemple des indicateurs prégnants. Sociologie du travail, 43(4), 533-551.

Boussard, V. (2008). Sociologie de la gestion. Les faiseurs de performance. Paris: Belin.

32 Par exemple le fait de ne pas interroger l'efficacité de la verbalisation et des pratiques de contrôle répressives. 
Boussard, V., \& Maugeri, S. (2003). Du politique dans les organisations. Sociologies des dispositifs de gestion. Paris: L'Harmattan.

Brosnan, P. (2005). La comptabilité de gestion : une technologie invisible. Travail et Emploi, 105, 720.

Clot, Y. (2001). Éditorial. Éducation permanente, 146.

Clot, Y. (2004). Action et connaissance en clinique de l'activité. Activités, 1(1), 23-33, http://www .activites.org/v1n1/vol1num1.book.pdf

Clot, Y. (2006). Clinique du travail et clinique de l'activité. Nouvelle Revue de psychosociologie, 1 , 165-177.

Clot, Y. (2008). Travail et pouvoir d'agir. Paris: P.U.F.

Clot, Y., \& Faïta, D. (2000). Genres et styles en analyse du travail. Concepts et méthodes. Travailler, 4, 7-42.

Clot, Y, Faïta, D., Fernandez, G., \& Scheller, L. (2000). Entretiens en autoconfrontation croisée : une méthode en clinique de l'activité. Pistes, 2(1).

Clot, Y, \& Litim, M. (2003). Sens du travail. In Allouche, J. (Ed.), Encyclopédie des Ressources Humaines (pp. 1534-1546). Paris: Vuibert.

Collard, D. (2010). De la stratégie orientée client aux « enquêtes client mystère ». Les « enquêtes client mystère » à la SNCF : une fiction aux effets pourtant bien réels ! Gérer et Comprendre, 102, 36-46.

Colville, I. (1981). Reconstructing "Behavioral Accounting“. Accounting, Organizations and Society, 6(2), 119-132.

Girin, J. (1986). L’objectivation des données subjectives : éléments pour une théorie du dispositif dans la recherche interactive. Colloque FNEGE-ISEOR, Lyon.

Girin, J. (1989). L'opportunisme méthodique dans les recherches sur la gestion des organisations. In CRG (Ed.), Mots croisés avec Jacques (pp. 10-20). Paris: CRG École polytechnique.

Honoré L. (2007). Approche discursive et implantation des outils RH : le cas de la mise en place des entretiens de progrès chez France Télécom Marine. Congrès de l'AGRH, Fribourg.

Kostulski, K., Clot, Y., Litim, M., \& Plateau, S. (2011). L'horizon incertain de la transformation en clinique de l'activité : une intervention dans le champ de l'éducation surveillée. Activités, $8(1)$, 129-145, http://www .activites.org/v8n1/v8n1.pdf

Lorino, P. (1995). Comptes et récits de la performance. Paris: Éditions d'Organisation.

Lorino, P. (2003a). Méthodes et pratiques de la performance (3ème édition). Paris: Éditions d'Organisation.

Lorino, P. (2003b). Vers une théorie pragmatique et sémiotique des outils appliquée aux instruments de gestion. In Le Moal P. \& Bourgeon L. (Eds.). Savoir gérer. Mélanges en l'honneur de JeanClaude Tarondeau (pp. 263-284). Paris: Vuibert.

Lorino, P. (2005). Théorie des organisations, sens et action : le cheminement historique, du rationalisme à la genèse instrumentale des organisations. In R. Teulier R. \& P. Lorino (Eds.), Entre connaissance et organisation : l'activité collective (pp. 54-71). Paris: La Découverte.

Moisdon, J.C. (1997). Du mode d'existence des outils de gestion. Paris: Seli Arslan.

Moisdon, J.C. (2000). Quelle est la valeur de ton point ISA ? Nouveaux outils de gestion et de régulation dans le système hospitalier français. Sociologie du travail, 42, 31-49.

Moisdon, J.-C. (2005). Comment apprend-on par les outils de gestion ? Retour sur une doctrine d'usage. In R. Teulier \& P. Lorino (Eds.), Entre connaissance et organisation : l'activité collective (pp. 239-250). Paris: La Découverte.

Moisdon, J.C. (2008). Règles de gestion, outils, organisation. Le Libellio d'AEGIS, 4(1), 18-27.

Nahapiet, J. (1988). The rhetoric and reality of an accounting change: a study of resource allocation. Accounting, Organizations and Society, 13(4), 333-358.

Riveline, C. (1981). Évaluation des coûts. Éléments d'une théorie de la gestion. Paris: École des Mines de Paris.

Simondon, G. (1958). Du mode d'existence des objets techniques. Paris: Aubier.

Suquet, J.B. (2003). Situations de fraude, gestion de la fraude : le cas de la STIVO. Paris: Mémoire de 
DEA de l'Université Paris X - Nanterre.

Suquet, J.B. (2008). Les dynamiques professionnelles de la relation de service. Le cas de la fraude sur le réseau de surface de la RATP. Paris: Thèse de doctorat de l'École Polytechnique.

\begin{abstract}
RESUME
À partir d'une recherche-intervention menée sur la transformation du contrôle sur le réseau de surface de la RATP (bus et tramway), suite à la mise en œuvre de la démarche «Bus Attitude», les auteurs décrivent et analysent le «genre du métier » de contrôleur. Ils montrent que certains indicateurs de gestion peuvent jouer un rôle structurant dans la constitution et le maintien du "genre professionnel » au point de le dévitaliser. Dès lors, ils s'interrogent sur le bien-fondé d'une action sur les indicateurs de gestion pour transformer les pratiques professionnelles et revitaliser le «genre professionnel », en mettant en évidence à la fois les vertus et les limites d'une telle action et en questionnant le rôle joué par le chercheur dans l'intervention.
\end{abstract}

\title{
MOTS CLES
}

indicateurs de gestion, «genre professionnel», rechercheintervention.

\section{REFERENCEMENT}

Collard, D., \& Suquet, J.-B. (2013). Indicateurs de gestion et difficultés de renouvellement $\mathrm{du}$ «genre professionnel». Vertus et limites d'une action sur les indicateurs pour transformer les pratiques professionnelles. Activités, 10(2), 131-156, http://www .activites.org/v10n2/v10n2.pdf

Article soumis le 28 janvier 2013, accepté pour publication le 6 juillet 2013. 
Annexe 1 : «l'escalier des modes d'action »

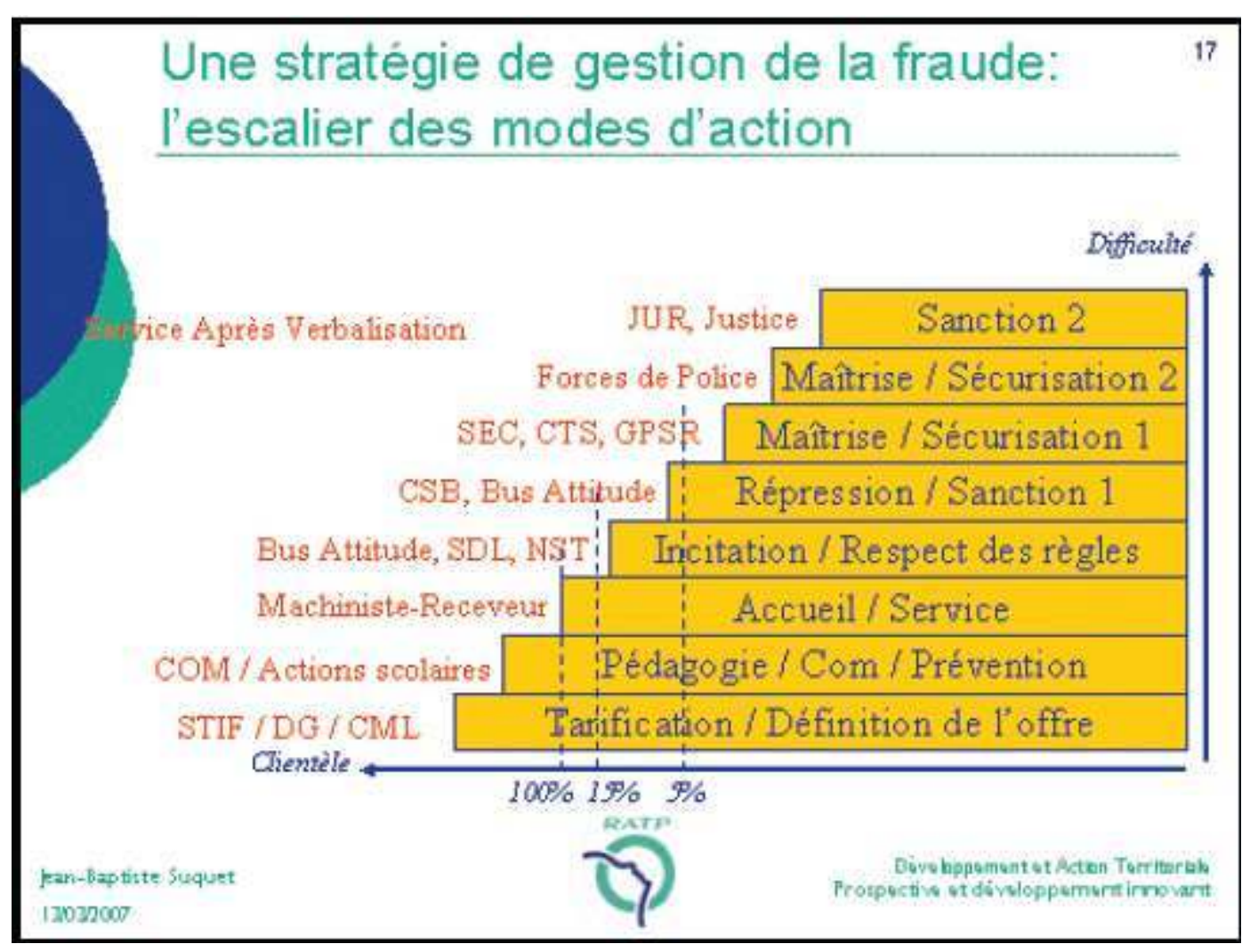


Annexe 2 : la « matrice des modes d'action »

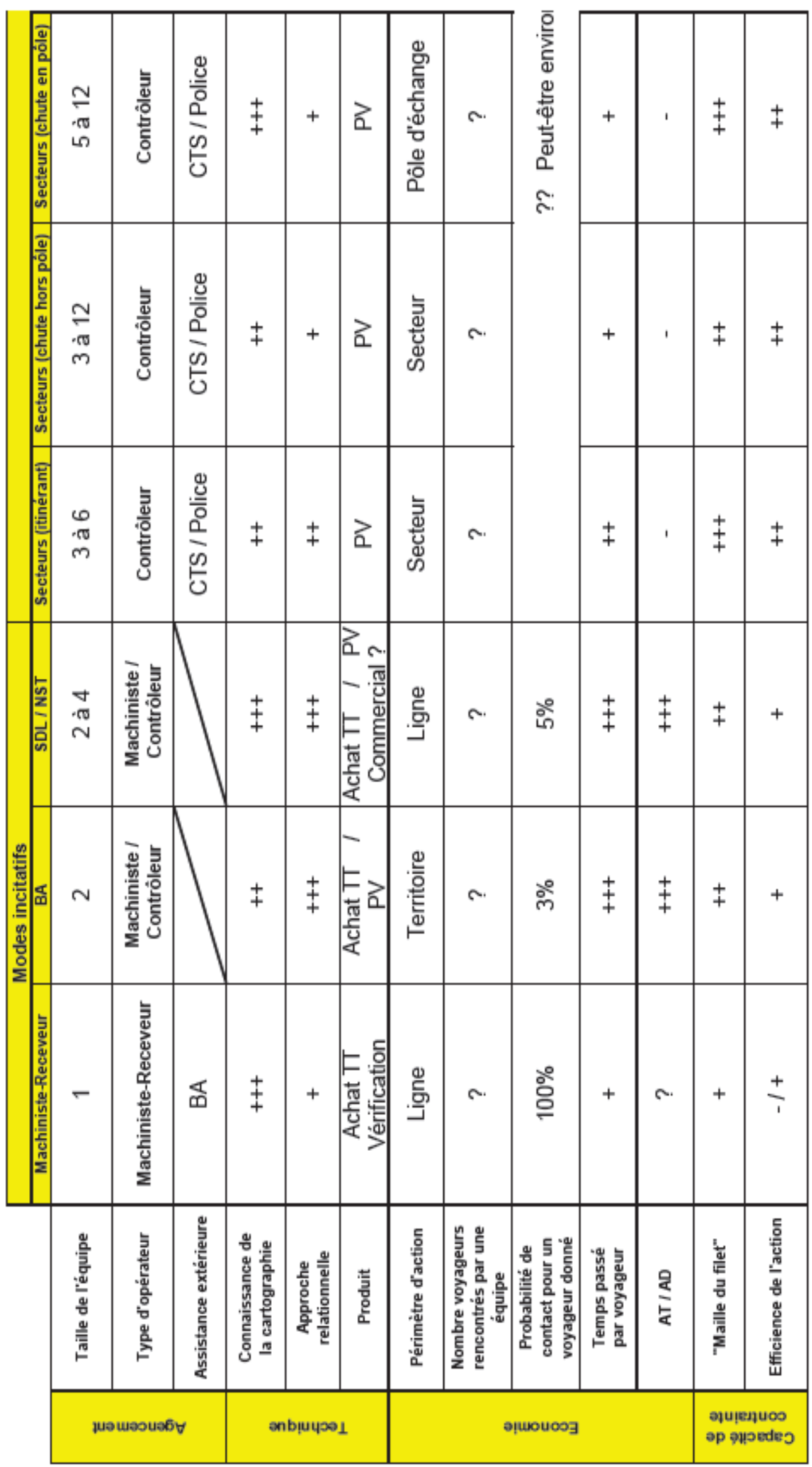

